\title{
Mindfulness, mindful eating e comer intuitivo na abordagem da obesidade e transtornos alimentares*
}

Marina Rodrigues Barbosa ${ }^{1,2}$

(D) https://orcid.org/0000-0001-7502-243X

Fernanda Rodrigues de Oliveira Penaforte ${ }^{3}$

(D) https://orcid.org/0000-0001-8483-1562

Ana Flavia de Sousa Silva ${ }^{4}$

(D) https://orcid.org/0000-0003-0000-8953
* Este artigo refere-se à chamada temática "Mindfulness e outras práticas contemplativas".

${ }_{1}$ Universidade Federal de Uberlândia, Faculdade de Medicina, Uberlândia, MG, Brasil.

2 Universidade de São Paulo, Escola de Enfermagem de Ribeirão Preto, Centro Colaborador da OPAS/OMS para o Desenvolvimento da Pesquisa em Enfermagem, Ribeirão Preto, SP, Brasil.

${ }^{3}$ Universidade Federal de Uberaba, Curso de Nutrição, Uberaba, MG, Brasil.

${ }^{4}$ Universidade de São Paulo, Faculdade de Medicina de Ribeirão Preto, Ribeirão Preto, SP, Brasil.

\begin{abstract}
Objetivo: realizar uma revisão integrativa da literatura acerca do papel das intervenções baseadas em mindfulness, mindful eating e comer intuitivo na abordagem do sobrepeso e obesidade e dos transtornos alimentares. Método: para esta revisão foram consultadas as bases PubMed, PePSIC, PsycINFO, LILACS, IBECS, SciELO e BVSPsi, e incluídos artigos publicados nos últimos 10 anos. Após o processo de análise dos estudos recuperados, 38 artigos compuseram o corpus final da revisão. Resultados: os estudos demonstraram os benefícios das abordagens centradas no mindfulness, mindful eating e comer intuitivo no comportamento alimentar e nos aspectos emocionais e psicológicos dos indivíduos com excesso de peso e transtorno alimentar, reduzindo o comer emocional, comer guiado por fatores externos, e episódios de binge eating; bem como reduzindo os níveis de estresse, ansiedade e depressão. Para a perda de peso, os resultados são menos consistentes, haja visto que diversos estudos não observaram diferença para este parâmetro. Conclusões: as intervenções centradas no mindfulness, mindful eating e comer intuitivo são promissoras para a abordagem do sobrepeso/obesidade e dos transtornos alimentares, especialmente no que tange ao comportamento alimentar e aos aspectos psicoemocionais.
\end{abstract}

Descritores: Atenção Plena; Comportamento Alimentar; Comer Intuitivo; Transtorno Alimentar; Terapia Cognitivo Comportamental; Intervenções Baseadas em Mindfulness.

\section{Como citar este artigo}

Barbosa MR, Penaforte FRO, Silva AFS. Mindfulness, mindful eating and intuitive eating in the approach to obesity and eating disorders. SMAD, Rev Eletrônica Saúde Mental Álcool Drog. 2020;16(3):118-135. doi: https://dx.doi.org/10.11606/issn.1806-6976.smad.2020.165262 


\section{Mindfulness, mindful eating and intuitive eating in the approach to obesity and eating disorders}

Objective: to examine and summarize studies of mindfulness, mindful eating and intuitive eating in the approach of overweight / obesity and eating disorders. Method: for this integrative review, PubMed, PePSIC, PsycINFO, LILACS, IBECS, SciELO and BVS-Psi databases were consulted, and published articles published in the last 10 years. After analyzing the recovered studies, 38 articles made up the final review corpus. Results: the studies demonstrated the benefits of approaches centered on mindfulness, mindful eating and intuitive eating on eating behavior and on the emotional and psychological aspects of overweight and eating disorders individuals, harmful or emotional, followed by external factors and compulsive eating; as well as levels of stress, anxiety and depression. For weight loss, the results are less consistent, since several studies have found no difference for this parameter. Conclusions: as selected focused on mindfulness, mindful eating and intuitive eating are promising for addressing obesity and obesity and eating disorders, especially with regard to eating behavior and psycho-emotional aspects.

Descriptors: Mindfulness; Eating Behavior; Cognitive Behavioral Therapy; Intuitive Eating; Eating Disorder; Mindfulness-Based Intervention.

\section{Atención plena, alimentación consciente y alimentación intuitiva en el abordaje del obesidad y de los trastornos alimentarios}

Objetivo: desarrollar una revisión integrativa de la literatura sobre el papel de las intervenciones basadas en el mindfulness, mindful eating y alimentación intuitiva en el abordaje del sobrepeso / obesidad y de los trastornos alimentarios. Método: para esta revisión, se consultaron las bases de datos PubMed, PePSIC, PsycINFO, LILACS, IBECS, SciELO y BVS-Psi, y se incluyeron artículos publicados en los últimos 10 años. Después de analizar los estudios recuperados, 38 artículos constituyeron el corpus final de la revisión. Resultados: los estudios han demostrado los beneficios las intervenciones basadas en el mindfulness, ME y alimentación intuitiva para el comportamiento alimentario y los aspectos emocionales y psicológicos de las personas con sobrepeso/obesidad y trastornos alimentarios, reduciendo la alimentación emocional, el comer basado en factores externos y en los episodios de binge eating; además de reducir los niveles de estrés, ansiedad y depresión. Para la pérdida de peso, los resultados son menos consistentes, dado que varios estudios no han encontrado diferencias para este parámetro con las intervenciones. Conclusiones: las intervenciones basadas en el mindfulness, mindful eating y alimentación intuitiva son prometedoras para el abordaje del sobrepeso/obesidad y de los trastornos alimentarios, especialmente con respecto al comportamiento alimentario y los aspectos psicoemocionales.

Descriptores: Conducta Alimentaria; Atención Plena; Terapia Cognitivo-Conductual; Alimentación Intuitiva; Trastorno Alimentario; Intervención Basada en la Atención Plena. 


\section{Introdução}

O excesso de peso corporal é um dos problemas de saúde pública mais desafiadores da atualidade. Apesar da sua crescente prevalência, e do vasto conhecimento sobre seus inúmeros desdobramentos negativos para a saúde ${ }^{(1)}$, o tratamento desta condição permanece de difícil adesão. Por consequência, atualmente muito tem se questionado sobre os princípios nos quais se centram as abordagens tradicionais para o excesso de peso, que comumente se apoiam quase que exclusivamente na premissa das dietas, com restrição quantitativa e/ ou qualitativa da ingestão de alimentos. Especula-se que os resultados insatisfatórios desta abordagem estejam principalmente relacionados a não promoção de mudanças em comportamentos alimentares disfuncionais ${ }^{(2-3)}$.

Por sua vez, os transtornos alimentares (TA), caracterizados principalmente pela perturbação persistente na alimentação ou no comportamento relacionado ao comer, resultando em ingestão excessiva ou evitação estrita de alimentos ${ }^{(4)}$, muito embora sejam considerados de baixa prevalência, também cursam com graus significativos de morbidade e mortalidade, além de grandes prejuízos pessoais e psicossociais(5).

A abordagem do excesso de peso e dos TA é complexa e de difícil manejo, especialmente por se tratar de condições que cursam com relações disfuncionais com o comer e a comida, e com comprometimento do funcionamento psicossocial do sujeito(6-7). Neste cenário, estratégias inovadoras empregadas na abordagem de comportamentos alimentares disfuncionais têm despontado, com destaque para o mindfulness, mindful eating e comer intuitivo. O mindfulness (ou atenção plena) é descrito como um estado de consciência que emerge por meio da atenção que se tem, de forma intencional, ao momento presente, sem julgamento. Por ser um estado de consciência, o mindfulness pode ser desenvolvido e treinado, por meio de práticas formais e informais ${ }^{(8)}$.

Dentro do mindfulness, com olhar especial para a atenção aos aspectos relacionados ao comer e a comida, emerge o mindful eating (ME) ou "comer com atenção plena". O ME preconiza que os sujeitos façam suas escolhas alimentares de forma consciente e atenta aos sinais físicos de fome e saciedade ${ }^{(9)}$. Outra premissa importante do ME é a atenção a toda experiência envolvida no comer, notando os efeitos da comida nos sentidos e nas sensações físicas e emocionais que ocorrem antes, durante e após a alimentação, com abertura e sem julgamentos ${ }^{(10-11)}$.

O comer intuitivo (CI) ou "alimentação intuitiva", apesar de ser menos conhecido, segue na mesma linha e geralmente é usado de forma intercambiável com o ME, especialmente por ambos preconizarem o comer guiado pela sinalização fisiológica de fome e saciedade ${ }^{(12)}$. O CI possui dez princípios, com destaque para a rejeição às dietas, permissão incondicional para comer; comer por razões físicas, e não emocionais; e confiança no corpo e na sinalização de fome e saciedade para determinar quando e quanto comer(13-15).

Por se tratar de abordagens inovadoras, a sistematização do conhecimento no que tange aos resultados destas abordagens para a saúde dos sujeitos com sobrepeso e obesidade, e com TA, são pouco numerosos, apesar de crescentes. Algumas revisões foram conduzidas com o objetivo de avaliar os efeitos de abordagens baseadas no mindfulness na perda de peso(16-17), no tratamento da obesidade e dos transtornos

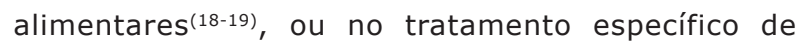
transtornos alimentares ${ }^{(14,20-21)}$; outras buscaram verificar os efeitos especificamente do mindful eating na perda de peso $^{(22)}$ ou na abordagem da obesidade e dos transtornos alimentares $^{(23)}$. Todavia, não foram encontradas revisões que reunissem os resultados de intervenções baseadas no mindfulness, mindful eating e comer intuitivo no comportamento alimentar, aspectos físicos e aspectos psicoemocionais em indivíduos com sobrepeso e obesidade, e com TA. Especificamente no cenário nacional, apenas um estudo de revisão integrativa foi encontrado na temática específica do mindful eating ${ }^{(23)}$. Portanto, este estudo teve por objetivo realizar uma revisão integrativa da literatura do crescente corpo de pesquisas que investigaram o papel das intervenções baseadas em mindfulness, mindful eating e comer intuitivo na abordagem do sobrepeso e da obesidade e dos TAs, especialmente no que diz respeito aos resultados no comportamento alimentar e nos aspectos físico e psicoemocionais, buscando contribuir na redução dessa lacuna do conhecimento. A sistematização desse conhecimento é importante para ampliar a compreensão acerca de sua aplicabilidade, especialmente para condições que cursam com comportamentos alimentares disfuncionais.

\section{Método}

Trata-se de uma revisão integrativa da literatura científica, que visa sintetizar e aprofundar o conhecimento acerca de um determinado assunto, buscando evidências consolidadas para a prática baseada em evidências (24-25).

Para esta revisão foram adotados procedimentos

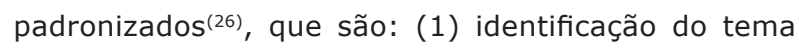
e da questão norteadora; (2) estabelecimento de critérios de inclusão/exclusão; (3) categorização dos estudos; (4) avaliação dos estudos; (5) interpretação dos resultados; (6) síntese do conhecimento(26). Adotou-se também o protocolo internacional para estudos de revisão sistemática e metanálises, o PRISMA (Preferred Reporting Items for Systematic Reviews 
and Meta-Analyses), para orientar tanto a inclusão e exclusão dos artigos da revisão, quanto a escrita do presente estudo(27).

Para a presente revisão, a questão norteadora foi definida a partir do método PICO, que prevê a definição do participante $(P)$, intervenção (I), comparação (C) e desfecho/outcomes $(\mathrm{O})^{(28)}$. O item comparação não foi empregado neste estudo, portanto o critério $C$ foi excluído da redação da pergunta. Esta alteração é prevista na metodologia PICO em alguns casos. A questão norteadora do estudo foi: Quais os resultados no comportamento alimentar, em aspectos físicos e em aspectos psicoemocionais ( $\mathrm{O}$ ) de abordagens centradas no mindfulness, mindful eating e comer intuitivo (I) em sujeitos com sobrepeso, obesidade e transtornos alimentares $(P)$ ?

\section{Percurso da seleção dos artigos}

A seleção dos artigos ocorreu entre outubro e dezembro de 2019. Foram realizadas buscas nas bases de dados PubMed, PePSIC, PsycINFO, Literatura Latinoamericana e do Caribe em Ciências da Saúde (LILACS), IBECS, Scientific Eletronic Library Online (SciELO) e BVS-Psi. Foram considerados trabalhos em português, inglês e espanhol. Estas bases de dados foram escolhidas por atingirem vasta literatura, no âmbito nacional e internacional, publicada sobre o tema de interesse, e também por incluir periódicos conceituados na área da saúde.

Para as buscas, foram utilizados descritores indexados segundo a padronização do DeCs/MeSh, nas suas versões em português e inglês. Os descritores indexados utilizados foram: transtorno alimentar (eating disorder ou feeding disorder), comportamento alimentar (eating behavior), atenção plena (Mindfulness), obesidade (obesity) e excesso de peso (overweight). Outros descritores não indexados, mas que se relacionam diretamente ao tema, também foram utilizados, sendo eles: mindful eating e comer intuitivo (intuitive eating). Estes unitermos apareceram em, pelo menos, um dos seguintes campos de busca: título, resumo, assunto ou palavra-chave.

Foram adotados os seguintes critérios para inclusão dos artigos: (a) artigos publicados entre janeiro de 2009 a dezembro de 2019; (b) estudos empíricos que utilizaram explícita e especificamente programas com abordagens centradas no mindfulness, mindful eating e comer intuitivo; (c) estudos feitos com adultos; (d) que estivessem disponíveis gratuitamente para leitura na íntegra e (e) publicados nos idiomas português, inglês e espanhol. Foram excluídos: (a) estudos com crianças e idosos; (b) materiais como monografias, editoriais, livros, capítulos de livros, resenhas e resumos em anais de congressos; (c) artigos de revisão de literatura; (d) artigos de casos clínicos; (e) estudos qualitativos; (f) estudos sobre elaboração e/ou validação de protocolos ou instrumentos científicos; $(\mathrm{g})$ estudos feitos com programas/técnicas de intervenção que utilizam alguns elementos do mindfulness, mindful eating e/ ou comer intuitivo, mas não são centrados nessas abordagens. Os estudos que se repetiram em mais de uma base de dados foram computados apenas uma vez.

Para verificar se artigos encontrados atendiam aos critérios de inclusão e exclusão, foram lidos seus respectivos títulos e resumos. Esse procedimento foi realizado por dois juízes independentes, ambos com familiaridade com tema. No caso de discordância, a análise era feita por um terceiro juiz, também com experiência na temática. Os artigos selecionados nesta primeira etapa foram recuperados para leitura na íntegra, sendo novamente aplicados os critérios de inclusão e exclusão nos textos completos. Os artigos que permaneceram, após a análise pela leitura na íntegra, compuseram o corpus final de análise do presente estudo.

Para a etapa de análise foi realizado um fichamento de todos os artigos que compuseram o corpus final, compilando as seguintes informações: título, autores, ano, local de publicação, método/tipo de estudo, amostra, objetivos e principais resultados. A análise na íntegra dos artigos permitiu a organização das categorias que visam responder à questão norteadora desta revisão.

\section{Resultados}

\section{Caracterização dos artigos}

As buscas nas bases de dados originaram um total de 785 artigos. A base com maior número de artigos foi a Pubmed, seguida por SciELO, IBECS e LILACS. A primeira etapa da análise gerou a exclusão de 40 artigos que estavam duplicados. Posteriormente, foram excluídos 528 artigos, especialmente por se tratarem de estudos de associação ou por abordarem o assunto de forma tangencial. Dos 54 artigos restantes para leitura na íntegra, 16 foram excluídos. Essas exclusões se deram, por exemplo, por tratarem-se de estudos que apenas descreviam um programa a ser implementado, ou por utilizaram intervenções que apresentavam apenas elementos pontuais do mindfulness, ME ou CI. Assim, um total de 38 artigos compuseram o corpus final desta revisão. A Figura 1 apresenta o fluxograma de estratégia de seleção dos estudos, de acordo com o protocolo PRISMA.

Os artigos que compõem a presente revisão são exclusivamente internacionais e no idioma inglês. O país com maior número de publicações foi os Estados Unidos $(n=27 ; 71,0 \%)$, seguido por Portugal $(n=03$; $7,9 \%)$, Inglaterra $(n=02 ; 5,2 \%)$, Austrália $(n=2 ; 5,2 \%)$, 
Holanda $(n=01 ; 2,6 \%)$, Canadá $(n=01 ; 2,6 \%)$, Nova Zelândia $(n=01 ; 2,6 \%)$ e Irã $(n=01 ; 2,6 \%)$. O ano com maior quantidade de publicação foi 2017 ( $n=09$; $23,7 \%)$, seguido por $2016(n=06 ; 15,8 \%)$. Os anos de 2010, 2014, 2018 e 2019 contribuíram com quatro estudos (10,8\%) em cada ano. Os anos de 2011 e 2013 foram os com menor número de publicações, contando com apenas 01 artigo $(2,7 \%)$ em cada ano.

O tamanho amostral dos estudos variou de 10

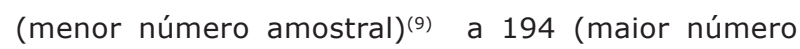
amostral) de participantes. As pesquisas que contaram com o maior número amostral(29-30) foram derivadas do mesmo protocolo de pesquisa. A maior parte dos estudos foi desenvolvida com homens e mulheres $(n=21$; $55,2 \%$ ), contudo uma parcela importante dos estudos foi realizada exclusivamente com mulheres $(n=17 ; 44,7)$.

Foi observado que a maioria dos estudos $(n=31$, $81,6 \%$ ) foi desenvolvida com indivíduos com excesso de peso, com 06 estudos (15,8\%) feitos com portadores de TA, e apenas $01(2,6 \%)$ realizado com mulheres com excesso de peso e binge eating. Estudos realizados com público com condições clínicas específicas foram pouco numerosos. Foram encontradas pesquisas com mulheres com histórico atual ou pregresso de câncer ( $n=03$, $7,9 \%)$, com gestantes ( $n=02,5,2 \%$ ) e com indivíduos em pós-operatório de cirurgia bariátrica $(n=01,2,6 \%)$.

No que tange ao tipo do estudo, a maioria dos artigos se enquadra no tipo randomizado $(n=21$;
$55,2 \%)$, seguido pela avaliação dos participantes antes e após a intervenção $(n=13 ; 34,2 \%)$. A intervenção mais longa durou 6 meses $^{(31)}$, e as mais curtas tiveram duração de um mês(32-33). O tempo de intervenção mais comumente encontrado nos estudos foi de 8 semanas $(n=08,21,0 \%)$, seguido pelas intervenções de 10 semanas $(n=07,18,4 \%)$. A maioria dos estudos utilizou a intervenção baseada no mindfulness ( $n=18 ; 47,3 \%)$, seguida pelas intervenções que utilizaram mindful eating $(n=11 ; 28,9 \%)$, mindfulness e mindful eating associadas $(n=5 ; 13,1 \%)$, e comer intuitivo $(n=04 ; 10,5 \%)$.

É importante mencionar também que a maior parte das pesquisas com grupo controle contaram com grupo controle ativo ( $n=20 ; 52,6 \%$ ), ou seja, os participantes receberam algum tipo de intervenção que não envolvia mindfulness, mindful eating e comer intuitivo. Apenas 03 estudos $(7,8 \%)$ não fizeram nenhum tipo de intervenção no grupo controle.

A seguir serão apresentados os resultados organizados a partir da leitura na íntegra dos estudos que compuseram o corpus desta revisão, objetivando responder à questão norteadora: (1) resultados das abordagens centradas no mindfulness, mindful eating e comer intuitivo no comportamento alimentar; (2) resultados das abordagens centradas no mindfulness, mindful eating e comer intuitivo em aspectos físicos e (3) resultados das abordagens centradas no mindfulness, mindful eating e comer intuitivo em aspectos psicoemocionais.

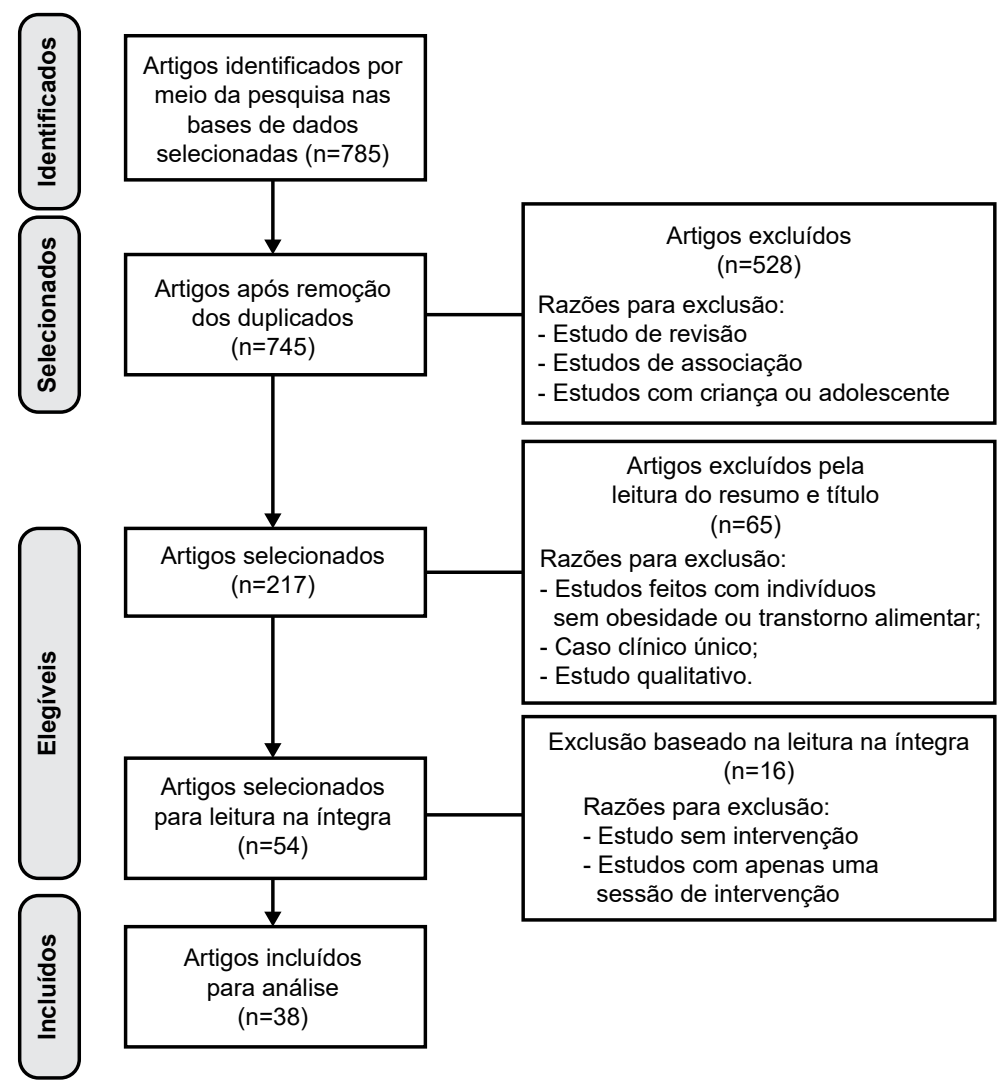

Figura 1 - Fluxograma do processo de seleção dos estudos, elaborado segundo recomendações do protocolo PRISMA(27) 
Categoria 1) Resultados das abordagens centradas no mindfulness, mindful eating e comer intuitivo no comportamento alimentar.

Os estudos com sujeitos com sobrepeso e obesidade que tiveram entre seus objetivos melhorias no comportamento alimentar $(55,3 \%)$ trazem resultados promissores. Com as intervenções apoiadas no mindfulness, ME ou CI foram observadas reduções nos níveis de food craving, no comer guiado por estímulos externos, no comer emocional, no comer guiado pela recompensa, nos episódios de compulsão alimentar e de binge eating comparados aos grupos controle. Para as intervenções com avaliação do tipo antes e depois, foram vistas melhorias na consciência alimentar, redução nos episódios de compulsão alimentar, aumentos nos escores de mindful eating e de comer intuitivo e melhoria na ingestão de frutas e verduras. Para os estudos feitos com portadores de TA $(18,4 \%)$ observou-se redução nos episódios de binge eating, redução nos escores do questionário Eating Attitudes Test (EAT), aumento nos níveis de CI e diminuição nos níveis de psicopatologia alimentar após a participação nas intervenções (Figuras 2 e 3).

Poucos estudos $(7,9 \%)$ não observaram diferenças entre os grupos experimental versus grupo controle para os parâmetros do comportamento alimentar avaliados. Como por exemplo, o estudo ${ }^{(34)}$ que identificou melhoria nos níveis de comer emocional, comer desinibido e sintomas de dependência alimentar em ambos os grupos de gestantes (intervenção e controle), sem diferença entre eles. É importante pontuar que o grupo controle, nesse estudo, também recebeu intervenção, denominada Emotional Brain Training (EBT), cujo objetivo foi treinar a autorregulação emocional dos participantes, com vistas a reduzir seus níveis de estresse ${ }^{(34)}$. Resultados semelhantes foram obtidos em um outro estudo(35), especialmente na redução do comer relacionado ao estresse ao final da intervenção, em todos os grupos, sem diferença entre eles. Também neste estudo todos os grupos receberam algum tipo de intervenção, conforme as combinações: (1) intervenção para redução do estresse baseado em mindfulness (programa Mindfulness Based Stress Reduction), (2) intervenção para redução do estresse baseada na inter-relação entre estresse, alimentação e bem-estar, apoiada na terapia cognitivo comportamental, e (3) uma combinação das intervenções dos grupos 1 e 2 (Figura 2 e 3).

\section{Categoria 2) Resultados das abordagens centradas no mindfulness, mindful eating e comer intuitivo em aspectos físicos.}

As pesquisas com intervenções com avaliação do tipo antes e depois, desenvolvidas com indivíduos com excesso de peso, obtiveram resultados satisfatórios no que diz respeito aos aspectos físicos, uma vez que a maioria $(66,7 \%)$ observou redução significativa no peso, no IMC e nos níveis de proteína $C$ reativa. Vale destacar também que a maior parte dos estudos randomizados $(72,7 \%)$, nos quais os participantes foram divididos em grupo intervenção (mindfulness e mindful eating) e grupo controle ativo (que recebiam algum tipo de intervenção), não encontrou diferenças significativas entre os grupos para a perda de peso. Entretanto, houve redução significativa na glicemia de jejum e na razão TG/HDL no grupo experimental relativo ao grupo

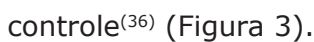

No que tange os estudos com portadores de TA, os resultados apontam para redução na preocupação com o peso, forma física e na supervalorização do peso após participação nas intervenções baseadas em mindfulness, comparados ao grupo controle. Também houve aumento na satisfação corporal, evidenciando os benefícios de tais intervenções para os participantes.

\section{Categoria 3) Resultados das abordagens centradas no mindfulness, mindful eating e comer intuitivo em aspectos psicoemocionais.}

Dentre os estudos realizados com indivíduos com sobrepeso e obesidade, $27,0 \%$ tiveram entre seus objetivos a avaliação dos impactos das intervenções baseadas em mindfulness nos aspectos emocionais e psicológicos, tais como depressão, ansiedade, estresse, flexibilidade psicológica e saúde mental geral. Estes estudos também trazem resultados promissores, pois $(80,0 \%)$ destes identificaram melhora dos parâmetros relativos ao sofrimento psíquico, depressão ou ansiedade ${ }^{(37-38)}$.

Os estudos indicam que após a intervenção baseada em mindfulness e mindful eating houve redução significativa dos níveis de depressão, ansiedade, estresse percebido e nível de cortisol(9,39-40). Somado a isso, alguns impactos positivos foram observados, tais como, melhora na capacidade de respostas às sensações corporais, flexibilidade psicológica e aceitação psicológica, também descrita como redução da evitação experiencial(41-42) (Figura 2).

Já nos estudos realizados com indivíduos com diagnóstico de TA, apenas $(5,2 \%)$ tiveram entre seus objetivos a avaliação dos impactos das intervenções baseadas em mindfulness nos aspectos emocionais e psicológicos. Um destes estudos foi o realizado com mulheres com $\mathrm{TA}^{(43)}$, com intervenção de 12 semanas integrando conceitos de psicoeducação e mindfulness, no qual foi observada redução significativa na inflexibilidade psicológica, vergonha externa e autocrítica; e aumento no engajamento com vida, na autocompaixão, nos níveis de autoconfiança e níveis de mindfulness. Importante 
ressaltar que esses ganhos foram mantidos em reavaliações 3 e 6 meses após intervenção.

Em outro trabalho, também realizado com mulheres com TA ${ }^{(33)}$, os autores realizaram intervenção baseada em mindfulness por quatro semanas e observaram redução nos sintomas de depressão e estresse, e melhora na flexibilidade psicológica em relação à imagem corporal, quando comparado ao grupo controle (Figura 2).

\begin{tabular}{|c|c|c|c|c|c|}
\hline $\begin{array}{l}\text { Título, autores } \\
\text { ano e país de } \\
\text { publicação }\end{array}$ & Tipo de estudo & $\begin{array}{l}\text { População avaliada } \\
\text { (tamanho para análise) }\end{array}$ & Tipo de Intervenção & $\begin{array}{c}\text { Duração e } \\
\text { componentes da } \\
\text { intervenção }\end{array}$ & $\begin{array}{c}\text { Práticas de mindfulness } \\
\text { presentes na } \\
\text { intervenção }\end{array}$ \\
\hline $\begin{array}{l}\text { Alberts et al. } \\
(2010) \\
\text { Holanda }^{(44)}\end{array}$ & $\begin{array}{l}\text { Estudo de intervenção } \\
\text { randomizado: grupo } \\
\text { controle e intervenção }\end{array}$ & $\begin{array}{l}\text { Adultos com obesidade } \\
(n=19)\end{array}$ & $\begin{array}{l}\text { Intervenção baseada } \\
\text { em mindfulness }\end{array}$ & $\begin{array}{l}7 \text { semanas. } \\
\text { Sessões grupais } \\
(1 \mathrm{~h} 30) \text { com prática } \\
\text { formal em casa }\end{array}$ & $\begin{array}{l}\text { Escaneamento corporal e } \\
\text { respiração sentada }\end{array}$ \\
\hline $\begin{array}{l}\text { Courbasson et } \\
\text { al (2010) } \\
\text { Canada }^{(45)}\end{array}$ & $\begin{array}{l}\text { Estudo de intervenção: } \\
\text { comparação antes e após }\end{array}$ & $\begin{array}{l}\text { Adultos com diagnóstico } \\
\text { de transtorno alimentar e } \\
\text { uso de substâncias } \\
(n=38)\end{array}$ & $\begin{array}{l}\text { Intervenção baseada } \\
\text { em mindfulness } \\
\text { associada à TCC* }\end{array}$ & $\begin{array}{l}16 \text { semanas. } \\
\text { Sessões grupais ( } 2 \\
\text { horas) com prática } \\
\text { formal em casa }\end{array}$ & $\begin{array}{l}\text { Prática de atenção plena } \\
\text { do não julgamento, } \\
\text { desenvolvimento da } \\
\text { confiança e manutenção } \\
\text { da mente aberta }\end{array}$ \\
\hline $\begin{array}{l}\text { Dalen et al } \\
(2010) \mathrm{EUA}^{(9)}\end{array}$ & $\begin{array}{l}\text { Estudo de intervenção } \\
\text { piloto: comparação antes } \\
\text { e após }\end{array}$ & $\begin{array}{l}\text { Adultos com obesidade } \\
(n=10)\end{array}$ & $\begin{array}{l}\text { Intervenção baseada } \\
\text { em mindfulness } \\
\text { criada para indivíduos } \\
\text { com obesidade e } \\
\text { sobrepeso }\left(\mathrm{MEAL}^{\dagger}\right)\end{array}$ & $\begin{array}{l}6 \text { semanas. } \\
\text { Sessões grupais } \\
\text { semanais ( } 2 \text { horas) } \\
\text { com prática formal } \\
\text { em casa }\end{array}$ & $\begin{array}{l}\text { Prática de atenção plena, } \\
\text { consciência às sensações } \\
\text { do corpo e exercício das } \\
\text { fomes }\end{array}$ \\
\hline $\begin{array}{l}\text { Hepworth } \\
(2010) \\
\text { Austrália(46) }^{(4)}\end{array}$ & $\begin{array}{l}\text { Estudo de intervenção } \\
\text { piloto: comparação antes } \\
\text { e após }\end{array}$ & $\begin{array}{l}\text { Mulheres com } \\
\text { diagnóstico de transtorno } \\
\text { alimentar } \\
(n=33)\end{array}$ & $\begin{array}{l}\text { Atendimento } \\
\text { ambulatorial, com } \\
\text { atividade grupal }\end{array}$ & $\begin{array}{l}10 \text { semanas. } \\
\text { Sessões grupais } \\
\text { (duração não } \\
\text { informada) com } \\
\text { prática formal em casa }\end{array}$ & $\begin{array}{l}\text { Respiração sentada } \\
\text { e prática do comer } \\
\text { consciente }\end{array}$ \\
\hline $\begin{array}{l}\text { Daubenmier } \\
\text { et al. (2011) } \\
\text { EUA }^{(39)}\end{array}$ & $\begin{array}{l}\text { Estudo clínico } \\
\text { randomizado piloto: grupo } \\
\text { controle e intervenção }\end{array}$ & $\begin{array}{l}\text { Mulheres com sobrepeso } \\
\text { e/ou obesidade }(n=47)\end{array}$ & $\begin{array}{l}\text { Intervenção baseada } \\
\text { nos protocolos MBSR }{ }^{\ddagger} ; \\
\text { MBCT }{ }^{\S} \text { e MB-EAT" }\end{array}$ & \begin{tabular}{|l|}
8 semanas. \\
Sessões grupais $(2,5$ \\
horas) e um retiro ( 7 \\
horas) com prática \\
formal diária e informal \\
( 6 dias/semana) \\
\end{tabular} & $\begin{array}{l}\text { Respiração sentada; } \\
\text { escaneamento corporal; } \\
\text { yoga; prática da bondade } \\
\text { amorosa; “3 minutos } \\
\text { para respirar; MEđ nas } \\
\text { refeições }\end{array}$ \\
\hline $\begin{array}{l}\text { Anglin (2012) } \\
\text { EUA }^{(47)}\end{array}$ & $\begin{array}{l}\text { Estudo clínico } \\
\text { randomizado: grupo } \\
\text { controle e intervenção }\end{array}$ & $\begin{array}{l}\text { Adultos com obesidade, } \\
\text { sedentários e sem } \\
\text { histórico de doenças } \\
\text { crônicas }(n=16)\end{array}$ & $\begin{array}{l}\text { Grupo intervenção: } \\
\text { baseada no comer } \\
\text { intuitivo; grupo } \\
\text { controle: restrição } \\
\text { calórica }\end{array}$ & 6 semanas & Não mencionou \\
\hline $\begin{array}{l}\text { Daubenmier } \\
\text { et al. (2012) } \\
\text { EUA }^{(38)}\end{array}$ & $\begin{array}{l}\text { Estudo clínico } \\
\text { randomizado piloto: grupo } \\
\text { controle e intervenção }\end{array}$ & $\begin{array}{l}\text { Mulheres com sobrepeso } \\
\text { e/ou obesidade }(n=47)\end{array}$ & $\begin{array}{l}\text { Intervenção baseada } \\
\text { nos protocolos } \\
\text { protocolos MBSR } \\
\text { MBCT§ e MB-EAT" }\end{array}$ & $\begin{array}{l}8 \text { semanas. } \\
\text { Sessões grupais } \\
\text { ( } 2,5 \text { horas) um } \\
\text { retiro ( } 7 \text { horas) com } \\
\text { prática formal diária } \\
\text { e informal ( } 6 \text { dias/ } \\
\text { semana) } \\
\end{array}$ & $\begin{array}{l}\text { Respiração sentada; } \\
\text { escaneamento corporal; } \\
\text { yoga; prática da bondade } \\
\text { amorosa; “3 minutos } \\
\text { para respirar; ME『 nas } \\
\text { refeições }\end{array}$ \\
\hline $\begin{array}{l}\text { Woolhouse } \\
\text { et al. (2012) } \\
\text { Austrália }^{(49)}\end{array}$ & $\begin{array}{l}\text { Estudo de intervenção } \\
\text { não randomizado: } \\
\text { comparação antes e após }\end{array}$ & $\begin{array}{l}\text { Mulheres com transtorno } \\
\text { alimentar } \\
(n=54)\end{array}$ & $\begin{array}{l}\text { Intervenção baseada } \\
\text { em mindfulness } \\
\text { com associação da } \\
\text { TCC* para transtorno } \\
\text { alimentar (Programa } \\
\text { MEG) }\end{array}$ & $\begin{array}{l}10 \text { semanas. } \\
\text { Sessão grupal ( } 3 \\
\text { horas), e uma sessão } \\
\text { de acompanhamento } \\
\text { após } 3 \text { meses. }\end{array}$ & $\begin{array}{l}\text { Escaneamento corporal; } \\
\text { respiração sentada; } \\
\text { prática do manejo dos } \\
\text { sentimentos relativos ao } \\
\text { alimento }\end{array}$ \\
\hline $\begin{array}{l}\text { Kidd et al. } \\
(2013) \text { EUA }^{(50)}\end{array}$ & $\begin{array}{l}\text { Estudo de intervenção: } \\
\text { comparação antes e após }\end{array}$ & $\begin{array}{l}\text { Mulheres com obesidade } \\
(n=12)\end{array}$ & $\begin{array}{l}\text { Intervenção baseada } \\
\text { em MEđ }\end{array}$ & $\begin{array}{l}8 \text { semanas. } \\
\text { Sessões grupais ( } 1 \text { - } \\
\text { 1h30). }\end{array}$ & $\begin{array}{l}\text { Consciência dos sentidos } \\
\text { e padrões } \\
\text { sem pressão para mudar; } \\
\text { sem julgamento }\end{array}$ \\
\hline $\begin{array}{l}\text { Corsica et al. } \\
(2014) \text { EUA }^{(35)}\end{array}$ & $\begin{array}{l}\text { Estudo clínico } \\
\text { randomizado: } 3 \text { grupos }\end{array}$ & $\begin{array}{l}\text { Mulheres }(98 \%) \text { com } \\
\text { excesso de peso } \\
(n=53)\end{array}$ & $\begin{array}{l}\text { Grupos: (1) } \\
\text { MBSR; (2) TCC*; } \\
\text { e (3) intervenção } \\
\text { combinada (1 e 2) }\end{array}$ & $\begin{array}{l}6 \text { semanas. } \\
\text { Sessões grupais } \\
\text { ( } 50 \text { a } 80 \text { minutos) } \\
\text { com prática formal e } \\
\text { informal em casa } \\
\end{array}$ & $\begin{array}{l}\text { Escaneamento corporal, } \\
\text { respiração sentada, yoga, } \\
\text { meditação da bondade } \\
\text { amorosa e prática } \\
\text { informal }\end{array}$ \\
\hline $\begin{array}{l}\text { Daubenmier } \\
\text { et al. (2014) } \\
\text { EUA }^{(40)}\end{array}$ & $\begin{array}{l}\text { Estudo clínico } \\
\text { randomizado piloto: grupo } \\
\text { controle e intervenção }\end{array}$ & $\begin{array}{l}\text { Mulheres com sobrepeso } \\
\text { e/ou obesidade }(n=47)\end{array}$ & $\begin{array}{l}\text { Intervenção baseada } \\
\text { nos protocolos MBSR }{ }^{\ddagger} ; \\
\text { MBCT }^{\S} \text { e MB-EAT" }\end{array}$ & $\begin{array}{l}8 \text { semanas. } \\
\text { Sessões grupais } \\
\text { ( } 2,5 \text { horas) e um } \\
\text { retiro ( } 7 \text { horas) com } \\
\text { prática formal diária } \\
\text { e informal (6 dias/ } \\
\text { semana) } \\
\end{array}$ & $\begin{array}{l}\text { Respiração sentada; } \\
\text { escaneamento corporal; } \\
\text { yoga; prática da bondade } \\
\text { amorosa; "3 minutos } \\
\text { para respirar; ME『 nas } \\
\text { refeições }\end{array}$ \\
\hline $\begin{array}{l}\text { Kristeller et al. } \\
(2014) \text { EUA }^{(11)}\end{array}$ & $\begin{array}{l}\text { Estudo clinico } \\
\text { randomizado: grupo } \\
\text { controle e intervenção }\end{array}$ & $\begin{array}{l}\text { Adultos com sobrepeso } \\
\text { ou obesidade }(n=150)\end{array}$ & $\begin{array}{l}\text { Grupos: (1) } \\
\text { intervenção MB-EAT"; } \\
\text { (2) psicoeducacional } \\
\text { e cognitivo- } \\
\text { comportamental; e (3) } \\
\text { grupo controle passivo }\end{array}$ & $\begin{array}{l}9 \text { semanas. } \\
\text { Sessões grupais } \\
(1 \mathrm{~h} 30-2 \mathrm{~h}) \text { e } 3 \text { reforços } \\
\text { mensais }\end{array}$ & $\begin{array}{l}\text { Respiração sentada; MEđ } \\
\text { nas refeições }\end{array}$ \\
\hline
\end{tabular}




\begin{tabular}{|c|c|c|c|c|c|}
\hline $\begin{array}{c}\text { Título, autores } \\
\text { ano e país de } \\
\text { publicação }\end{array}$ & Tipo de estudo & $\begin{array}{l}\text { População avaliada } \\
\text { (tamanho para análise) }\end{array}$ & Tipo de Intervenção & $\begin{array}{c}\text { Duração e } \\
\text { componentes da } \\
\text { intervenção }\end{array}$ & $\begin{array}{c}\text { Práticas de mindfulness } \\
\text { presentes na } \\
\text { intervenção }\end{array}$ \\
\hline $\begin{array}{l}\text { Mantzios et } \\
\text { al. (2014) } \\
\text { Inglaterra(51) }\end{array}$ & $\begin{array}{l}\text { Estudo clínico } \\
\text { randomizado: grupo } \\
\text { controle e intervenção }\end{array}$ & $\begin{array}{l}\text { Adultos com sobrepeso } \\
\text { e/ou obesidade }(n=152)\end{array}$ & $\begin{array}{l}\text { Mindful Attention } \\
\text { and Awareness em } \\
\text { ambos os grupos, um } \\
\text { realizado em grupo } \\
\text { e o outro com prática } \\
\text { individual }\end{array}$ & $\begin{array}{l}6 \text { semanas. } \\
\text { Sessões grupais ( } 2 \\
\text { horas) e individuais, } \\
\text { com prática formal } \\
\text { em casa }\end{array}$ & $\begin{array}{l}\text { Prática da respiração } \\
\text { sentada e escaneamento } \\
\text { corporal }\end{array}$ \\
\hline $\begin{array}{l}\text { Asadollahi et } \\
\text { al. (2015) } \\
\text { Irã }{ }^{(52)}\end{array}$ & $\begin{array}{l}\text { Estudo clínico } \\
\text { randomizado: com } 4 \\
\text { grupos }\end{array}$ & $\begin{array}{l}\text { Adultos com obesidade } \\
(n=60)\end{array}$ & $\begin{array}{l}\text { Grupos: (1) MBCT\&; (2) } \\
\text { orientação nutricional; } \\
\text { (3) associação (1 e 2) } \\
\text { e (4) grupo controle } \\
\text { passivo }\end{array}$ & $\begin{array}{l}8 \text { semanas. } \\
\text { Sessões grupais ( } 2 \\
\text { horas) com pratica } \\
\text { formal em casa }\end{array}$ & $\begin{array}{l}\text { Meditação; “3 minutos } \\
\text { de respiração"; } \\
\text { escaneamento corporal; } \\
\text { checagem do estômago } \\
\text { nas refeições }\end{array}$ \\
\hline $\begin{array}{l}\text { Stites et al. } \\
(2015) \text { EUA }^{(32)}\end{array}$ & $\begin{array}{l}\text { Estudo clínico } \\
\text { randomizado: grupo } \\
\text { intervenção e intervenção } \\
\text { tardia }\end{array}$ & $\begin{array}{l}\text { Adultos com sobrepeso } \\
\text { e/ou obesidade }(n=28)\end{array}$ & $\begin{array}{l}\text { Intervenção baseada } \\
\text { em ME匹 }\end{array}$ & $\begin{array}{l}4 \text { semanas. } \\
\text { Sessões grupais } \\
(1 \mathrm{~h} 30)\end{array}$ & Não mencionado \\
\hline $\begin{array}{l}\text { Boucher et al. } \\
\text { (2016) Nova } \\
\text { Zelândia( }^{(42)}\end{array}$ & $\begin{array}{l}\text { Estudo de intervenção } \\
\text { piloto: comparação antes } \\
\text { e após }\end{array}$ & $\begin{array}{l}\text { Mulheres com sobrepeso } \\
(n=40)\end{array}$ & \begin{tabular}{|l|} 
Intervenção baseada \\
na alimentação \\
intuitiva, associada à \\
abordagem psicológica \\
\end{tabular} & $\begin{array}{l}14 \text { semanas. } \\
12 \text { sessões grupais } \\
(15-20 \text { minutos })\end{array}$ & Não mencionado \\
\hline $\begin{array}{l}\text { Chacko et al. } \\
(2016) \text { EUA }^{(53)}\end{array}$ & $\begin{array}{l}\text { Estudo clínico } \\
\text { randomizado: grupo } \\
\text { controle e intervenção }\end{array}$ & $\begin{array}{l}\text { Adultos pós cirurgia } \\
\text { bariátrica (1 a } 5 \text { anos) } \\
\text { ( } n=18)\end{array}$ & $\begin{array}{l}\text { Intervenção baseada } \\
\text { nos protocolos MBSR }{ }^{\ddagger} \text {, } \\
\text { MB-EATIl e Mindful } \\
\text { Self-compassion } \\
\text { (MSC) }\end{array}$ & $\begin{array}{l}10 \text { semanas. } \\
\text { Sessões grupais } \\
\text { (1h30) e um retiro (4 } \\
\text { horas) com prática } \\
\text { formal em casa }\end{array}$ & $\begin{array}{l}\text { Respiração sentada, } \\
\text { prática da bondade } \\
\text { amorosa, escaneamento } \\
\text { corporal e yoga } \\
\text { consciente }\end{array}$ \\
\hline $\begin{array}{l}\text { Chung et al. } \\
(2016) \\
\text { EUA }^{(54)}\end{array}$ & $\begin{array}{l}\text { Estudo de intervenção: } \\
\text { comparação antes e após }\end{array}$ & $\begin{array}{l}\text { Mulheres com sobrepeso } \\
\text { e histórico de câncer } \\
\text { de mama (tratamento } \\
\text { finalizado a, pelo menos, } \\
3 \text { meses) } \\
(n=22)\end{array}$ & \begin{tabular}{|l|} 
Intervenção baseada \\
em ME ${ }^{\Uparrow}$ associada à \\
orientação dietética \\
para perda de peso
\end{tabular} & $\begin{array}{l}12 \text { semanas. } \\
\text { Sessões grupais por } 6 \\
\text { semanas }\end{array}$ & $\begin{array}{l}\text { Respiração sentada; } \\
\text { ME匹 nas refeições; } \\
\text { escaneamento corporal } \\
\text { e mindfulness em } \\
\text { movimento }\end{array}$ \\
\hline $\begin{array}{l}\text { Daubenmier } \\
\text { et al. }(2016 \\
\text { EUA }^{(36)}\end{array}$ & $\begin{array}{l}\text { Estudo clínico } \\
\text { randomizado: grupo } \\
\text { controle e intervenção }\end{array}$ & $\begin{array}{l}\text { Adultos com obesidade } \\
(n=194)\end{array}$ & \begin{tabular}{|l|} 
Intervenção baseada \\
nos protocolos MBSR ${ }^{\ddagger}$, \\
MBCT§ e MB-EAT"
\end{tabular} & $\begin{array}{l}12 \text { semanas. } \\
16 \text { sessões grupais } \\
(2-2 \mathrm{~h} 30), \text { com prática } \\
\text { formal e informal em } \\
\text { casa }\end{array}$ & $\begin{array}{l}\text { Respiração sentada; } \\
\text { caminhando atentamente; } \\
\text { bondade amorosa; yoga e } \\
\text { MEInas refeições }\end{array}$ \\
\hline $\begin{array}{l}\text { Lucas et al. } \\
(2016) \text { EUA }^{(55)}\end{array}$ & $\begin{array}{l}\text { Estudo de intervenção: } \\
\text { comparação antes e após }\end{array}$ & $\begin{array}{l}\text { Mulheres com sobrepeso } \\
\text { e/ou obesidade e } \\
\text { histórico de câncer } \\
(\mathrm{n}=17)\end{array}$ & $\begin{array}{l}\text { Intervenção baseada } \\
\text { em mindfulness e } \\
\text { orientação dietética }\end{array}$ & 8 semanas & $\begin{array}{l}\text { Prática da respiração } \\
\text { sentada e da yoga } \\
\text { consciente }\end{array}$ \\
\hline $\begin{array}{l}\text { Mason et al. } \\
(2016) \text { EUA }^{(29)}\end{array}$ & $\begin{array}{l}\text { Estudo clínico } \\
\text { randomizado: grupo } \\
\text { controle e intervenção }\end{array}$ & $\begin{array}{l}\text { Adultos com sobrepeso } \\
(\mathrm{n}=194)\end{array}$ & $\begin{array}{l}\text { Grupo intervenção: } \\
\text { dieta/exercícios e } \\
\text { mindfulness. Grupo } \\
\text { controle: dieta e } \\
\text { exercício físico } \\
\end{array}$ & $\begin{array}{l}12 \text { semanas } \\
16 \text { sessões grupais } \\
(2-2 \mathrm{~h} 30), \text { com prática } \\
\text { formal e informal em } \\
\text { casa }\end{array}$ & $\begin{array}{l}\text { Respiração sentada; } \\
\text { caminhando atentamente; } \\
\text { bondade amorosa e yoga }\end{array}$ \\
\hline $\begin{array}{l}\text { Mason et al. } \\
(2016) \text { EUA }^{(56)}\end{array}$ & $\begin{array}{l}\text { Estudo clínico } \\
\text { randomizado: grupo } \\
\text { controle e intervenção }\end{array}$ & $\begin{array}{l}\text { Adultos com obesidade } \\
(n=194)\end{array}$ & $\begin{array}{l}\text { Intervenção baseada } \\
\text { em mindfulness. } \\
\text { Grupo controle e } \\
\text { intervenção receberam } \\
\text { orientação nutricional } \\
\text { e de prática de } \\
\text { exercício. (SHINE } \\
\text { clinical trial) }\end{array}$ & $\begin{array}{l}12 \text { semanas. } \\
16 \text { sessões grupais } \\
(2-2 \mathrm{~h} 30), \text { com prática } \\
\text { formal em casa. }\end{array}$ & $\begin{array}{l}\text { Respiração sentada; } \\
\text { caminhando atentamente; } \\
\text { bondade amorosa e yoga }\end{array}$ \\
\hline $\begin{array}{l}\text { Pinto Gouveia } \\
(2016) \\
\text { Portugal }{ }^{(43)}\end{array}$ & $\begin{array}{l}\text { Estudo de intervenção: } \\
\text { comparação antes e após }\end{array}$ & $\begin{array}{l}\text { Mulheres com transtorno } \\
\text { alimentar } \\
\text { e sobrepeso }(n=31)\end{array}$ & $\begin{array}{l}\text { O BEfree é um } \\
\text { programa psicológico } \\
\text { com estratégias de } \\
\text { psicoeducação e } \\
\text { novas abordagens } \\
\text { contextual- } \\
\text { comportamentais, } \\
\text { como mindfulness, } \\
\text { autocompaixão } \\
\end{array}$ & $\begin{array}{l}12 \text { semanas. } \\
\text { Sessões grupais } \\
(2 \mathrm{~h} 30)\end{array}$ & $\begin{array}{l}\text { Respiração sentada e } \\
\text { escaneamento corporal }\end{array}$ \\
\hline $\begin{array}{l}\text { Adler et } \\
\text { al.(2017) } \\
\text { EUA }^{(30)}\end{array}$ & $\begin{array}{l}\text { Estudo clínico } \\
\text { randomizado: grupo } \\
\text { controle e intervenção }\end{array}$ & $\begin{array}{l}\text { Adultos com obesidade } \\
(n=194)\end{array}$ & $\begin{array}{l}\text { Intervenção baseada } \\
\text { em mindfulness. } \\
\text { Grupo controle e } \\
\text { intervenção receberam } \\
\text { orientação nutricional } \\
\text { e de prática de } \\
\text { exercício. (SHINE } \\
\text { clinical trial) }\end{array}$ & $\begin{array}{l}12 \text { semanas. } \\
16 \text { sessões grupais } \\
(2-2 \mathrm{~h} 30)\end{array}$ & $\begin{array}{l}\text { Respiração sentada; } \\
\text { caminhando atentamente; } \\
\text { bondade amorosa e yoga }\end{array}$ \\
\hline $\begin{array}{l}\text { Carpenter et al. } \\
\text { (2017) EUA }{ }^{(57)}\end{array}$ & $\begin{array}{l}\text { Estudo clínico } \\
\text { randomizado piloto: grupo } \\
\text { controle e intervenção }\end{array}$ & $\begin{array}{l}\text { Adultos com sobrepeso } \\
(n=75)\end{array}$ & $\begin{array}{l}\text { Intervenção baseada } \\
\text { em mindfulness. } \\
\text { Grupo controle e } \\
\text { intervenção receberam } \\
\text { orientação sobre } \\
\text { perda de peso } \\
\end{array}$ & $\begin{array}{l}11 \text { semanas. } \\
\text { Intervenção por } \\
\text { telefone (semanal, } \\
\text { com prática de } 60 \\
\text { segundos) }\end{array}$ & $\begin{array}{l}\text { Prática da respiração } \\
\text { sentada e MEđ }\end{array}$ \\
\hline
\end{tabular}




\begin{tabular}{|c|c|c|c|c|c|}
\hline $\begin{array}{l}\text { Título, autores } \\
\text { ano e país de } \\
\text { publicação }\end{array}$ & Tipo de estudo & $\begin{array}{l}\text { População avaliada } \\
\text { (tamanho para análise) }\end{array}$ & Tipo de Intervenção & $\begin{array}{c}\text { Duração e } \\
\text { componentes da } \\
\text { intervenção }\end{array}$ & $\begin{array}{c}\text { Práticas de mindfulness } \\
\text { presentes na } \\
\text { intervenção }\end{array}$ \\
\hline $\begin{array}{l}\text { Duarte et } \\
\text { al. }(2017) \\
\text { Portugal( }{ }^{(33)}\end{array}$ & $\begin{array}{l}\text { Estudo clínico } \\
\text { randomizado piloto: grupo } \\
\text { controle e intervenção }\end{array}$ & $\begin{array}{l}\text { Mulheres com transtorno } \\
\text { alimentar } \\
(n=20)\end{array}$ & $\begin{array}{l}\text { Intervenção breve } \\
\text { baseada em } \\
\text { mindfulness e } \\
\text { compaixão }\end{array}$ & $\begin{array}{l}4 \text { semanas. } \\
\text { Uma sessão grupal } \\
(2 \mathrm{~h} 30) \text { e } 3 \text { semanas } \\
\text { de prática individual } \\
\text { guiada por áudio }\end{array}$ & $\begin{array}{l}\text { Respiração sentada; } \\
\text { escaneamento } \\
\text { corporal; ME"le imagens } \\
\text { compassivas }\end{array}$ \\
\hline $\begin{array}{l}\text { Palmeira et } \\
\text { al. }(2017) \\
\text { Portugal }{ }^{(37)}\end{array}$ & $\begin{array}{l}\text { Estudo clínico } \\
\text { randomizado: grupo } \\
\text { controle e intervenção }\end{array}$ & $\begin{array}{l}\text { Mulheres com sobrepeso } \\
\text { e/ou obesidade }(n=52)\end{array}$ & $\begin{array}{l}\text { Intervenção baseada } \\
\text { em MB-EAT॥, terapia } \\
\text { de aceitação e } \\
\text { compaixão }\end{array}$ & $\begin{array}{l}10 \text { semanas. } \\
\text { Sessões grupais } \\
(2 \mathrm{~h} 30) \text { e mais } 2 \\
\text { sessões de reforço a } \\
\text { cada } 3,5 \text { meses, com } \\
\text { prática formal em casa }\end{array}$ & $\begin{array}{l}\text { Prática da bondade } \\
\text { amorosa, ME`e } \\
\text { respiração sentada }\end{array}$ \\
\hline $\begin{array}{l}\text { Raja-Khan } \\
\text { et al. (2017) } \\
\text { EUA }^{(38)}\end{array}$ & $\begin{array}{l}\text { Estudo clínico } \\
\text { randomizado: grupo } \\
\text { controle e intervenção }\end{array}$ & $\begin{array}{l}\text { Mulheres com sobrepeso } \\
\text { e/ou obesidade }(n=86)\end{array}$ & MBSR $\ddagger$ & $\begin{array}{l}8 \text { semanas. } \\
\text { Sessões grupais } \\
\text { (2h30) e um retiro (6 } \\
\text { horas), com prática } \\
\text { formal em casa }\end{array}$ & Não mencionado \\
\hline $\begin{array}{l}\text { Richards et } \\
\text { al.(2017) } \\
\text { EUA }^{(58)}\end{array}$ & $\begin{array}{l}\text { Estudo de intervenção } \\
\text { piloto: comparação antes } \\
\text { e após }\end{array}$ & $\begin{array}{l}\text { Mulheres com transtorno } \\
\text { alimentar } \\
(n=120)\end{array}$ & $\begin{array}{l}\text { Programa baseado } \\
\text { nos } 10 \text { princípios do } \\
\text { Comer Intuitivo }\end{array}$ & $\begin{array}{l}10 \text { semanas. } \\
\text { Sessões individuais }\end{array}$ & Não mencionado \\
\hline $\begin{array}{l}\text { Spadaro et al. } \\
(2017) \text { EUA }^{(31)}\end{array}$ & $\begin{array}{l}\text { Estudo clínico } \\
\text { randomizado piloto: grupo } \\
\text { controle e intervenção }\end{array}$ & $\begin{array}{l}\text { Adultos com sobrepeso } \\
(n=46)\end{array}$ & $\begin{array}{l}\text { Intervenção baseado } \\
\text { no protocolo MBSR }{ }^{\ddagger} \text { e } \\
\text { MB-EAT॥ associada à } \\
\text { orientação de redução } \\
\text { calórica e prática de } \\
\text { exercício físico }\end{array}$ & $\begin{array}{l}6 \text { meses. } \\
\text { Sessões grupais }(1 \\
-1 \mathrm{~h} 30)\end{array}$ & $\begin{array}{l}\text { Escaneamento corporal; } \\
\text { respiração sentada; } \\
\text { check-in de três minutos } \\
\text { e bondade amorosa e } \\
\text { MEđ }\end{array}$ \\
\hline $\begin{array}{l}\text { Kristeller et al. } \\
(2018) \text { EUA }^{(59)}\end{array}$ & $\begin{array}{l}\text { Estudo clínico } \\
\text { randomizado: grupo } \\
\text { controle e intervenção }\end{array}$ & $\begin{array}{l}\text { Adultos com obesidade } \\
(n=117)\end{array}$ & $\begin{array}{l}\text { Grupo intervenção } \\
\text { MB-EAT\| }\end{array}$ & $\begin{array}{l}10 \text { semanas. } \\
\text { Sessões grupais } \\
\text { ( } 2 \text { h } 30) \text { com } 2 \text { sessões } \\
\text { de reforço ( } 2 \text { horas). }\end{array}$ & $\begin{array}{l}\text { Respiração sentada; } \\
\text { ME匹 nas refeições; } \\
\text { escaneamento corporal e } \\
\text { prática do perdão }\end{array}$ \\
\hline $\begin{array}{l}\text { Laraia et } \\
\text { al.(2018) } \\
\text { EUA }^{(34)}\end{array}$ & $\begin{array}{l}\text { Estudo clínico não } \\
\text { randomizado: grupo } \\
\text { controle e intervenção }\end{array}$ & $\begin{array}{l}\text { Gestantes (12-19 } \\
\text { semanas gestacionais) } \\
\text { com sobrepeso e/ou } \\
\text { obesidade }(n=46)\end{array}$ & $\begin{array}{l}\text { Intervenção baseada } \\
\text { no MBSR }{ }^{\ddagger} ; \text { MBCT }^{\S}, \text { e } \\
\text { MB-EAT" }\end{array}$ & $\begin{array}{l}8 \text { semanas. } \\
\text { Sessões grupais ( } 2 \\
\text { horas) }\end{array}$ & $\begin{array}{l}\text { Respiração sentada; } \\
\text { bondade amorosa e da } \\
\text { autocompaixão }\end{array}$ \\
\hline $\begin{array}{l}\text { Mason et al. } \\
(2018) \text { EUA }^{(60)}\end{array}$ & $\begin{array}{l}\text { Estudo de intervenção: } \\
\text { comparação antes e após }\end{array}$ & $\begin{array}{l}\text { Adultos com sobrepeso } \\
(n=104)\end{array}$ & $\begin{array}{l}\text { Intervenção baseada } \\
\text { em ME匹 }\end{array}$ & $\begin{array}{l}28 \text { dias. } \\
\text { Sessão individual } \\
\text { (5-10 minutos) com } \\
\text { ligação telefônica } \\
\text { diária }\end{array}$ & Respiração sentada; ME匹 \\
\hline $\begin{array}{l}\text { Vieten et al. } \\
(2018) \text { EUA }^{(41)}\end{array}$ & $\begin{array}{l}\text { Estudo de intervenção: } \\
\text { comparação antes e após }\end{array}$ & $\begin{array}{l}\text { Gestantes ( } 8 \text { - } 20 \\
\text { semanas gestacionais) } \\
\text { com sobrepeso }(n=110)\end{array}$ & $\begin{array}{l}\text { Intervenção baseada } \\
\text { no MBSR }{ }^{\ddagger} ; \mathrm{MBCT}^{\S} \mathrm{e} \\
\text { MB-EAT\| }\end{array}$ & $\begin{array}{l}8 \text { semanas. } \\
\text { Sessões grupais (2 } \\
\text { horas), com prática } \\
\text { formal em casa }\end{array}$ & $\begin{array}{l}\text { Respiração sentada; } \\
\text { mindfulness em } \\
\text { movimento; bondade } \\
\text { amorosa e escaneamento } \\
\text { corporal. }\end{array}$ \\
\hline $\begin{array}{l}\text { Cole et al. } \\
(2019) \text { EUA }^{(61)}\end{array}$ & $\begin{array}{l}\text { Estudo de intervenção } \\
\text { piloto: comparação antes } \\
\text { e após }\end{array}$ & $\begin{array}{l}\text { Adultos com sobrepeso } \\
\text { e/ou obesidade }(n=56)\end{array}$ & $\begin{array}{l}\text { Intervenção baseada } \\
\text { nos princípios do } \\
\text { comer intuitivo } \\
\text { (Programa My Body } \\
\text { Knows When - } \\
\text { MBKW) }\end{array}$ & $\begin{array}{l}10 \text { semanas. } \\
\text { Sessões grupais, } \\
\text { online e presencial } \\
(1 \mathrm{~h} 30)\end{array}$ & Não mencionado \\
\hline $\begin{array}{l}\text { Hanson et al. } \\
\text { (2019) Reino } \\
\text { Unido }^{(62)}\end{array}$ & $\begin{array}{l}\text { Estudo clinico } \\
\text { randomizado piloto: grupo } \\
\text { controle e intervenção }\end{array}$ & $\begin{array}{l}\text { Adultos com obesidade } \\
\text { grau } 3(n=33)\end{array}$ & $\begin{array}{l}\text { Intervenção baseada } \\
\text { em mindfulness. } \\
\text { Grupo controle: } \\
\text { indivíduos que não } \\
\text { completaram a } \\
\text { intervenção }\end{array}$ & $\begin{array}{l}8 \text { semanas. } \\
\text { Sessões grupais } \\
\text { a cada } 2 \text { semanas } \\
(1 \mathrm{~h} 30)\end{array}$ & Não mencionado \\
\hline $\begin{array}{l}\text { Stice et al. } \\
\text { (2019) EUA }\end{array}$ & $\begin{array}{l}\text { Estudo clínico } \\
\text { randomizado: grupo } \\
\text { controle e intervenção }\end{array}$ & $\begin{array}{l}\text { Mulheres com transtorno } \\
\text { alimentar } \\
(n=84)\end{array}$ & $\begin{array}{l}\text { Intervenção baseada } \\
\text { em mindfulness. } \\
\text { Grupo controle foi } \\
\text { Body Project Eating } \\
\text { Disorder - BPT }\end{array}$ & $\begin{array}{l}8 \text { semanas. } \\
\text { Sessões grupais ( } 1 \\
\text { hora) }\end{array}$ & Não mencionado \\
\hline $\begin{array}{l}\text { Thomas et al. } \\
(2019) \text { EUA }^{(64)}\end{array}$ & $\begin{array}{l}\text { Estudo clínico } \\
\text { randomizado: grupo } \\
\text { controle e intervenção }\end{array}$ & $\begin{array}{l}\text { Mulheres com sobrepeso } \\
\text { e/ou obesidade e } \\
\text { histórico de câncer (em } \\
\text { remissão ou ativo) } \\
(n=51)\end{array}$ & $\begin{array}{l}\text { Intervenção baseada } \\
\text { em mindfulness } \\
\text { associada a elementos } \\
\text { da TCC* e princípios } \\
\text { da psicologia } \\
\text { positiva. Os dois } \\
\text { grupos receberam } \\
\text { aconselhamento } \\
\text { nutricional }\end{array}$ & $\begin{array}{l}10 \text { semanas. } \\
\text { Sessões grupais } \\
\text { (1h30), com prática } \\
\text { formal em casa }\end{array}$ & Não mencionado \\
\hline
\end{tabular}

*TCC = Terapia Cognitivo Comportamental; ${ }^{+}$MEAL = Mindful Eating and Living $;{ }^{\ddagger}$ MBSR = Mindfulness - Based Stress Reduction; ${ }^{5}$ MBCT $=$ Mindfulness-Based Cognitive Therapy; "MB-EAT = Mindfulness-Based Eating Awareness Training; "ME = Mindful Eating

Figura 2 - Resumo da análise dos artigos incluídos: descrição da amostra, tipo e duração da intervenção utilizada, Ribeirão Preto, SP, Brasil, 2019 


\begin{tabular}{|c|c|c|c|}
\hline $\begin{array}{l}\text { Título, autores ano e } \\
\text { local de publicação }\end{array}$ & Instrumentos aplicados & Desfechos / parâmetros avaliados & Resultados encontrados \\
\hline $\begin{array}{l}\text { Alberts et al. (2010) } \\
\text { Holanda }^{(44)}\end{array}$ & $\begin{array}{l}\text { General Food Craving Questionnaire } \\
\text { Trait }\end{array}$ & $\begin{array}{l}\text { (a) peso; (b) desejos de comida; (c) } \\
\text { horas de prática diária. }\end{array}$ & $\begin{array}{l}\text { Participantes do grupo experimental } \\
\text { relataram desejos por comida } \\
\text { significativamente mais baixos após a } \\
\text { intervenção }\end{array}$ \\
\hline $\begin{array}{l}\text { Courbasson et al } \\
\text { (2010) } \\
\text { Canada }^{(45)}\end{array}$ & $\begin{array}{l}\text { Structured Clinical Interview for DSM-IV } \\
\text { disorders; Eating Disorder Examination } \\
\text { Questionnaire; BDI* }\end{array}$ & $\begin{array}{l}\text { (a) número de episódios de compulsão } \\
\text { alimentar; } \\
\text { (b) número de dias de compulsão } \\
\text { alimentar }\end{array}$ & $\begin{array}{l}\text { Redução do número de episódios } \\
\text { de compulsão alimentar relatados. } \\
\text { Redução das preocupações sobre } \\
\text { forma, peso e alimentação ao longo do } \\
\text { tempo. }\end{array}$ \\
\hline $\begin{array}{l}\text { Dalen et al (2010) } \\
\text { EUA }^{(9)}\end{array}$ & $\begin{array}{l}\mathrm{BES}^{\dagger} ; \\
\text { BDI*;PSS§; TFEQ"; KIMS"; Beck Anxiety } \\
\text { Inventory }\end{array}$ & $\begin{array}{l}\text { (a) alterações de peso e IMC‡; (b) } \\
\text { comportamento alimentar; (c) estresse } \\
\text { psicológico; (d) marcadores fisiológicos } \\
\text { de estresse }\end{array}$ & $\begin{array}{l}\text { Aumento nas medidas de consciência } \\
\text { em torno do comer, e redução de peso, } \\
\text { compulsão alimentar e depressão. } \\
\text { Houve redução da Proteína C-Reativa }\end{array}$ \\
\hline $\begin{array}{l}\text { Hepworth (2010) } \\
\text { Austrália }^{(46)}\end{array}$ & $\mathrm{EAT}^{* \star}-26$ & Sintomas alimentares desordenados & $\begin{array}{l}\text { Foram encontradas reduções em todas } \\
\text { as suBEScalas do EAT }{ }^{\star *}-26\end{array}$ \\
\hline $\begin{array}{l}\text { Daubenmier et al. } \\
\text { (2011) } E \cup A^{(39)}\end{array}$ & $\mathrm{KIMS}^{\pi} ; \mathrm{DEBQ}{ }^{\dagger+}$ & $\begin{array}{l}\text { (a) nível de atenção plena, (b) } \\
\text { comportamento alimentar, (c) peso, } \\
\text { gordura abdominal e (d) resposta ao } \\
\text { cortisol }\end{array}$ & $\begin{array}{l}\text { Aumentou na atenção e capacidade } \\
\text { de resposta às sensações corporais, } \\
\text { reduzindo a ansiedade e o comer } \\
\text { emocional. }\end{array}$ \\
\hline Anglin (2012) EUA ${ }^{(47)}$ & Não aplicou instrumento específico & $\begin{array}{l}\text { (a) peso; (b) circunferência da cintura; } \\
\text { (c) e IMC }\end{array}$ & $\begin{array}{l}\text { Houve perda ou manutenção do peso } \\
\text { nos dois grupos. A perda de peso total } \\
\text { foi maior no grupo restrição calórica }\end{array}$ \\
\hline $\begin{array}{l}\text { Daubenmier et al. } \\
\text { (2012) } \mathrm{EUA}^{(38)}\end{array}$ & $\begin{array}{l}\mathrm{PSS}^{\S} ; \quad \mathrm{DEBQ}^{\dagger+} ; \quad \mathrm{KIMS}^{\pi} \text { e; Block Food } \\
\text { Frequency; State-Trait Anxiety Scale- } \\
\text { Trait Form; }\end{array}$ & $\begin{array}{l}\text { (a) sofrimento psicológico; (b) } \\
\text { comportamento alimentar; (c) } \\
\text { parâmetros metabólicos (d) atividade da } \\
\text { telomerase }\end{array}$ & $\begin{array}{l}\text { Ambos os grupos tiveram aumento da } \\
\text { atividade da telomerase }\end{array}$ \\
\hline $\begin{array}{l}\text { Woolhouse et al. } \\
\text { (2012) Austrália } \\
\end{array}$ & $\begin{array}{l}\text { DEBQ't; Eating Disorders Inventory-3 } \\
\text { Symptom Checklist; Multifactorial } \\
\text { Assessment of Eating Disorders Scale; } \\
\text { Eating Self-Efficacy Scale; Emotional } \\
\text { Overeating Questionnaire; Cognitive } \\
\text { and Affective Mindfulness Scale- } \\
\text { Revised }\end{array}$ & $\begin{array}{l}\text { Frequência e gravidade dos eventos de } \\
\text { compulsão alimentar; }\end{array}$ & $\begin{array}{l}\text { Redução significativa na frequência e } \\
\text { gravidade da compulsão alimentar e } \\
\text { insatisfação com a imagem corporal }\end{array}$ \\
\hline $\begin{array}{l}\text { Kidd et al. (2013) } \\
\text { EUA }^{(50)}\end{array}$ & $\begin{array}{l}\text { 20-item Weight Efficacy Lifestyle } \\
\text { Questionnaire; 28-Mindful Eating } \\
\text { Questionnaire (MEQ); 20-item Center } \\
\text { for Epidemiologic Studies-Depression } \\
\text { Scale }\end{array}$ & $\begin{array}{l}\text { (a) sintomas depressivos; (b) peso, } \\
\text { altura, composição corporal, pressão } \\
\text { arterial, (c) estilo de vida; (d) nível de } \\
\text { ME¥+ }\end{array}$ & $\begin{array}{l}\text { Houve aumento da autoeficácia para } \\
\text { perda de peso e hábitos alimentares. }\end{array}$ \\
\hline $\begin{array}{l}\text { Corsica et al. (2014) } \\
\text { EUA( }^{(35)}\end{array}$ & $\begin{array}{l}\text { PSS\$-10; Eating and Appraisal Due to } \\
\text { Emotions and Stress Questionnaire; } \\
\text { Emotion- and Stress-Related Eating } \\
\text { subscale }\end{array}$ & $\begin{array}{l}\text { (a) estresse percebido; (b) alimentação } \\
\text { relacionada ao estresse e (c) perda de } \\
\text { peso }\end{array}$ & $\begin{array}{l}\text { A intervenção combinada resultou } \\
\text { em maiores reduções do estresse } \\
\text { percebido e produziu perda de peso a } \\
\text { curto prazo. }\end{array}$ \\
\hline $\begin{array}{l}\text { Daubenmier et al. } \\
\text { (2014) EUA }\end{array}$ & $\begin{array}{l}\text { DEBQ }{ }^{\dagger+} \text {; BES }{ }^{\dagger} ; \text { Body Responsiveness } \\
\text { Questionnaire }\end{array}$ & $\begin{array}{l}\text { (a) nível de atenção plena, (b) comer } \\
\text { emocional (c) peso, gordura abdominal } \\
\text { e (d) cortisol }\end{array}$ & $\begin{array}{l}\text { Maiores níveis de cortisol foram } \\
\text { relacionados a uma maior ingestão } \\
\text { alimentar emocional e restritiva, e a } \\
\text { uma menor consciência interoceptiva. }\end{array}$ \\
\hline $\begin{array}{l}\text { Kristeller et al. (2014) } \\
\text { EUA }^{(11)}\end{array}$ & $\begin{array}{l}\text { BES†; TFEQ"l; Power of Food } \\
\text { Scale;Rosenberg Self-Esteem }\end{array}$ & $\begin{array}{l}\text { (a) compulsão alimentar; (b) ingestão de } \\
\text { alimentos; (c) desejo por determinado } \\
\text { tipo de alimento; (d) depressão; (e) } \\
\text { autoestima; (f) e IMC } \text { IM }^{\ddagger}\end{array}$ & $\begin{array}{l}\text { Após a intervenção, } 95 \% \text { dos indivíduos } \\
\text { do grupo mindful eating deixaram de } \\
\text { atender aos critérios de transtorno } \\
\text { alimentar; contra } 76 \% \text { recebendo a } \\
\text { outra intervenção } \\
\end{array}$ \\
\hline $\begin{array}{l}\text { Mantzios et al. (2014) } \\
\text { Inglaterra(51) }\end{array}$ & $\begin{array}{l}\text { Mindful Attention and Awareness Scale; } \\
\text { Barratt Impulsivity Scale; Cognitive- } \\
\text { Behavioral Avoidance Scale; }\end{array}$ & $\begin{array}{l}\text { (a) peso; (b) atenção e consciência; (c) } \\
\text { impulsividade. }\end{array}$ & $\begin{array}{l}\text { A intervenção grupal produziu maior } \\
\text { perda de peso. A intervenção individual } \\
\text { aumentou os índices de atenção plena } \\
\text { e reduziu a impulsividade por alimentos. }\end{array}$ \\
\hline $\begin{array}{l}\text { Asadollahi et al. } \\
(2015) \\
\text { Irã }\end{array}$ & SCL $\$ \S_{-} 90-r$ & $\begin{array}{l}\text { (a) sintomas psiquiátricos; (b) e IMC } \neq \text {, } \\
\text { peso e altura }\end{array}$ & $\begin{array}{l}\text { A intervenção combinada teve um } \\
\text { impacto maior na perda de peso } \\
\text { dos participantes em comparação } \\
\text { à realização das intervenções } \\
\text { isoladamente. }\end{array}$ \\
\hline $\begin{array}{l}\text { Stites et al. (2015) } \\
\text { EUA }^{(32)}\end{array}$ & MEQ|IIII & $\begin{array}{l}\text { (a) peso e IMC } \text {; }^{\text {; }} \text { (b) parâmetros } \\
\text { metabólicos }\end{array}$ & $\begin{array}{l}\text { A intervenção produziu redução no } \\
\text { consumo de calorias e gordura nos } \\
\text { almoços em comparação com a } \\
\text { intervenção tardia }\end{array}$ \\
\hline $\begin{array}{l}\text { Boucher et al. (2016) } \\
\text { Nova Zelândia }^{(42)}\end{array}$ & $\begin{array}{l}\text { Intuitive Eating Scale (IES-1); Disorder } \\
\text { Examination-Screening Version } \\
\text { (EDE-S); Acceptance and Action } \\
\text { Questionnaire-II; Short Form 12-item } \\
\text { Health Survey }\end{array}$ & $\begin{array}{l}\text { (a) IMC‡; (b) alimentação intuitiva; (c) } \\
\text { compulsão alimentar; (d) flexibilidade } \\
\text { psicológica; (d) saúde mental. }\end{array}$ & $\begin{array}{l}\text { Aumento da pontuação nas escalas do } \\
\text { comer intuitivo, flexibilidade psicológica } \\
\text { e saúde mental. E redução na } \\
\text { compulsão alimentar. }\end{array}$ \\
\hline $\begin{array}{l}\text { Chacko et al. (2016) } \\
\text { EUA(53) }^{(53)}\end{array}$ & TFEQ"I-R1; Quality of life (QOL) & $\begin{array}{l}\text { (a) peso; (b) comportamento alimentar; } \\
\text { (c) compulsão alimentar; (d) parâmetros } \\
\text { metabólicos }\end{array}$ & $\begin{array}{l}\text { A intervenção foi eficaz na redução do } \\
\text { comer emocional. }\end{array}$ \\
\hline
\end{tabular}

(a Figura 3 continua na próxima página) 


\begin{tabular}{|c|c|c|c|}
\hline $\begin{array}{l}\text { Título, autores ano e } \\
\text { local de publicação }\end{array}$ & Instrumentos aplicados & Desfechos / parâmetros avaliados & Resultados encontrados \\
\hline $\begin{array}{l}\text { Chung et al. (2016) } \\
\text { EUA }^{(54)}\end{array}$ & MEQIIII & $\begin{array}{l}\text { (a) peso e e IMC‡; (b) níveis de mindful } \\
\text { eating }\end{array}$ & $\begin{array}{l}\text { Aumento significativo no nível de } \\
\text { mindful eating e redução do peso e e } \\
\text { IMC }\end{array}$ \\
\hline $\begin{array}{l}\text { Daubenmier et al. } \\
\text { (2016 EUA }{ }^{(36)}\end{array}$ & $\begin{array}{l}\text { 9-item Reward-based Eating Drive; } \\
\text { PSS }-10 ;\end{array}$ & $\begin{array}{l}\text { (a) Peso e altura, (b) pressão arterial; } \\
\text { (c) circunferência da cintura; (d) perfil } \\
\text { lipidêmico glicemia de jejum }\end{array}$ & $\begin{array}{l}\text { Melhora dos parâmetros de glicemia e } \\
\text { perfil lipidêmico do grupo intervenção } \\
\text { aos } 12 \text { e } 18 \text { meses. }\end{array}$ \\
\hline $\begin{array}{l}\text { Lucas et al. (2016) } \\
\text { USA }^{(55)}\end{array}$ & $\begin{array}{l}\text { Five Facet Mindfulness Questionnaire } \\
\text { (FFMQ); Mindfulness Attention } \\
\text { Awareness Scale;( MAAS); Food } \\
\text { Habits Questionnaire); Physical Activity } \\
\text { Questionnaire; Physical Performance } \\
\text { Battery; Pittsburg Sleep Quality Index. }\end{array}$ & $\begin{array}{l}\text { (a) nível de atenção plena; (b) } \\
\text { qualidade da dieta; (c) qualidade de } \\
\text { vida; (d) atividade física; (e) sono }\end{array}$ & $\begin{array}{l}\text { Houve aumento no nível de atenção } \\
\text { plena, consumo de frutas e vegetais, na } \\
\text { qualidade do sono após a intervenção. }\end{array}$ \\
\hline $\begin{array}{l}\text { Mason et al. (2016) } \\
\text { EUA(29) }^{(29)}\end{array}$ & $\begin{array}{l}\text { PSS§-10; 9-item Reward-based Eating } \\
\text { Drive (RED) scale }\end{array}$ & $\begin{array}{l}\text { (a) perda de peso; (b) mecanismos de } \\
\text { recompensa com a comida }\end{array}$ & $\begin{array}{l}\text { Grupo mindfulness apresentou niveis } \\
\text { mais baixos para a escala RED. }\end{array}$ \\
\hline $\begin{array}{l}\text { Mason et al. (2016) } \\
\text { EUA }^{(56)}\end{array}$ & MEQIIII & $\begin{array}{l}\text { (a) nível de mindfulness; (b) consumo } \\
\text { de doces; (c) glicemia de jejum }\end{array}$ & $\begin{array}{l}\text { Grupo intervenção apresentou maiores } \\
\text { aumentos nos níveis de Mindfulness } \\
\text { imediatamente após e manteve } \\
12 \text { meses depois. Grupo controle } \\
\text { apresentou aumento no consumo de } \\
\text { doces entre } 6 \text { e } 12 \text { meses após }\end{array}$ \\
\hline $\begin{array}{l}\text { Pinto Gouveia (2016) } \\
\text { Portugal(43) }\end{array}$ & $\begin{array}{l}\text { BES'; FFMQ'in; Acceptance and Action } \\
\text { Questionnaire-II; Cognitive Fusion } \\
\text { Questionnaire-Body } \\
\text { Image; Engage Living Scale; Forms of } \\
\text { Self-Criticising/Attacking \& Self- } \\
\text { Reassuring Scale; Self-Compassion } \\
\text { Scale }\end{array}$ & $\begin{array}{l}\text { (a) comportamento e atitudes } \\
\text { alimentares desordenadas; (b) } \\
\text { inflexibilidade psicológica; }\end{array}$ & $\begin{array}{l}\text { Redução da compulsão alimentar e } \\
\text { psicopatologia alimentar, inflexibilidade } \\
\text { psicológica, fusão cognitiva da } \\
\text { imagem corporal, vergonha externa e } \\
\text { autocrítica. } \\
\text { Houve aumento no engajamento } \\
\text { com vida valorizada, níveis de } \\
\text { autocompaixão, confiança e de } \\
\text { mindfulness. }\end{array}$ \\
\hline $\begin{array}{l}\text { Adler et al.(2017) } \\
\text { EUA }^{(30)}\end{array}$ & $\begin{array}{l}\text { FFMQ IIT e Pittsburgh Sleep Quality } \\
\text { Index }\end{array}$ & $\begin{array}{l}\text { (a) qualidade do sono; (b) nível de } \\
\text { mindfulness }\end{array}$ & $\begin{array}{l}\text { Ambos os grupos apresentaram } \\
\text { melhora da qualidade do sono, } \\
\text { especialmente com os maiores escores } \\
\text { no FFMQ. }\end{array}$ \\
\hline $\begin{array}{l}\text { Carpenter et al. } \\
\text { (2017) EUA }{ }^{(57)}\end{array}$ & $\begin{array}{l}\text { BES'; MEQIII!; FFMQ-Short Form; } \\
\text { Patient Health Questionnaire-2. }\end{array}$ & $\begin{array}{l}\text { (a) comportamento alimentar; (b) nível } \\
\text { de mindfulness; (c) nível de mindful } \\
\text { eating; (d) peso }\end{array}$ & $\begin{array}{l}\text { Melhora dos escores para mindful } \\
\text { eating, comportamento alimentar, } \\
\text { aceitação e flexibilidade psicológica } \\
\text { relacionada ao peso. }\end{array}$ \\
\hline $\begin{array}{l}\text { Duarte et al. }(2017) \\
\text { Portugal|(33) }\end{array}$ & $\begin{array}{l}\text { BES }+ \text {; FFMQ } \$ \text { Tा; Body Image Shame } \\
\text { Scale; 21 } \square \text { item Depression, Anxiety, } \\
\text { and Stress Scale; Cognitive Fusion } \\
\text { Questionnaire for food craving; } \\
\text { Body Image Acceptance and Action } \\
\text { Questionnaire; } 22 \square \text { item Forms of Self } \square \\
\text { Criticism and Self } \square \text { Reassurance Scale; }\end{array}$ & $\begin{array}{l}\text { (a) comportamentos transtornados; } \\
\text { (b) imagem corporal; (c) sintomas de } \\
\text { depressão e estresse; (d) e IMC }\end{array}$ & $\begin{array}{l}\text { A intervenção reduziu os sintomas } \\
\text { de transtorno alimentar, depressão e } \\
\text { estresse, bem como dos indicadores } \\
\text { de psicopatologia, supervalorização do } \\
\text { peso e da forma. Houve melhora da } \\
\text { flexibilidade psicológica em relação à } \\
\text { imagem corporal. }\end{array}$ \\
\hline $\begin{array}{l}\text { Palmeira et al. (2017) } \\
\text { Portugal|(37) }^{(37)}\end{array}$ & $\begin{array}{l}\text { Weight self-stigma Questionnaire; } \\
\text { OBESity Related Well-Being } \\
\text { Questionnaire; OBESity Related Well- } \\
\text { Being Questionnaire }\end{array}$ & $\begin{array}{l}\text { (a) comer emocional; (b) auto } \\
\text { estigma com a imagem corporal; (c) } \\
\text { comportamento alimentar; (d) IMC, } \\
\text { circunferência da cintura e niveis de } \\
\text { colesterol }\end{array}$ & $\begin{array}{l}\text { Grupo intervenção apresentou } \\
\text { diminuição significativa na autoestima } \\
\text { do peso, alimentação emocional e } \\
\text { descontrolada e aumento da qualidade } \\
\text { de vida vs controle. }\end{array}$ \\
\hline $\begin{array}{l}\text { Raja-Khan et al. } \\
(2017) \text { EUA }^{(38)}\end{array}$ & $\begin{array}{l}\text { Toronto Mindfulness Scale; Perceived } \\
\text { Stress Scale-10; Short Form-36; Brief } \\
\text { Symptoms Inventory-18. }\end{array}$ & $\begin{array}{l}\text { Parâmetros bioquímicos: glicose, } \\
\text { insulina, Proteína C-Reativa, } \\
\text { Hemoglobina glicada e cortisol. }\end{array}$ & $\begin{array}{l}\text { O grupo intervenção apresentou } \\
\text { redução significativa do estresse } \\
\text { percebido, da glicemia de jejum após } 8 \\
\text { e } 16 \text { semanas versus baseline, o que } \\
\text { não foi visto para o controle }\end{array}$ \\
\hline $\begin{array}{l}\text { Richards et al.(2017) } \\
\text { EUA }^{(58)}\end{array}$ & $\begin{array}{l}\text { "TIES; EAT }{ }^{* *} ; \text { Body Shape } \\
\text { Questionnaire; Outcome Questionnaire } \\
\text { (OQ). }\end{array}$ & $\begin{array}{l}\text { (a) comportamento alimentar e comer } \\
\text { intuitivo; (b) percepção da imagem } \\
\text { corporal; (c) peso; (d) }\end{array}$ & $\begin{array}{l}\text { Houve melhora nos escores do EAT**, } \\
\text { "TIIES e Body Shape Questionnaire. }\end{array}$ \\
\hline $\begin{array}{l}\text { Spadaro et al. (2017) } \\
\text { EUA }^{(31)}\end{array}$ & $\begin{array}{l}\text { MAAS**; Eating Behavior Inventory; } \\
\text { Block Food Frequency Questionnaire; } \\
\text { Paffenbarger Habits }\end{array}$ & $\begin{array}{l}\text { (a) peso; (b) comportamento e ingestão } \\
\text { alimentar: (c) nível de mindfulness }\end{array}$ & $\begin{array}{l}\text { Ambos os grupos tiveram perda de } \\
\text { peso, redução da ingestão alimentar } \\
\text { e aumento dos níveis de restrição } \\
\text { alimentar. Os resultados foram mais } \\
\text { intensos no grupo intervenção. O comer } \\
\text { desinibido e a percepção de fome } \\
\text { reduziram em ambos os grupos pós- } \\
\text { intervenção, sem diferença entre eles. }\end{array}$ \\
\hline $\begin{array}{l}\text { Kristeller et al. (2018) } \\
\text { EUA }^{(59)}\end{array}$ & $\begin{array}{l}\mathrm{BES}^{+} \text {; BD।**-II; FFMQ }{ }^{* \pi} ; \text { Functional } \\
\text { Assessment of Chronic Illness Therapy } \\
\text { - Spiritual Well-Being Scale }\end{array}$ & $\begin{array}{l}\text { (a) sintomas de transtorno alimentar; (b) } \\
\text { sintomas de ansiedade e depressão }\end{array}$ & $\begin{array}{l}\text { Os scores de ansiedade, depressão } \\
\text { e transtorno alimentar diminuíram no } \\
\text { grupo mindful eating. }\end{array}$ \\
\hline $\begin{array}{l}\text { Laraia et al.(2018) } \\
\text { EUA }^{(34)}\end{array}$ & $\begin{array}{l}\text { Cohen's Perceived Stress Scale; } \\
\text { DEBQ }+ \text { +; Pregnancy-Related Anxiety } \\
\text { Scale; Patient Health. }\end{array}$ & $\begin{array}{l}\text { (a) estresse percebido; (b) } \\
\text { comportamento alimentar; (c) sintomas } \\
\text { de depressão e ansiedade. }\end{array}$ & $\begin{array}{l}\text { Redução no estresse, ansiedade } \\
\text { relacionada a gravidez e depressão, } \\
\text { e no comer emocional em ambos os } \\
\text { grupos. }\end{array}$ \\
\hline
\end{tabular}

(a Figura 3 continua na próxima página) 


\begin{tabular}{|c|c|c|c|}
\hline $\begin{array}{l}\text { Título, autores ano e } \\
\text { local de publicação }\end{array}$ & Instrumentos aplicados & Desfechos / parâmetros avaliados & Resultados encontrados \\
\hline $\begin{array}{l}\text { Mason et al. (2018) } \\
\text { EUAA(60) }^{(6)}\end{array}$ & $\begin{array}{l}\text { Food Craving Questionnaire - Trait - } \\
\text { Reduced; Reward-based Eating Drive } \\
\text { Scale; Palatable Eating Motives Scale. }\end{array}$ & $\begin{array}{l}\text { (a) comer guiado pela recompensa; } \\
\text { (b) razões para comer alimentos } \\
\text { palatáveis; (c) engajamento com as } \\
\text { práticas; (d) peso }\end{array}$ & $\begin{array}{l}\text { Houve redução no comer relacionado } \\
\text { à recompensa e no comer por razões } \\
\text { sociais. }\end{array}$ \\
\hline $\begin{array}{l}\text { Vieten et al. (2018) } \\
\text { EUA }^{(41)}\end{array}$ & $\begin{array}{l}\text { FFMQ }{ }^{\text {TIT; }} \text { MEQIIII; PSS ; Acceptance } \\
\text { and Action Questionnaire; Emotion } \\
\text { Regulation Questionnaire; Patient } \\
\text { Health Questionnaire. }\end{array}$ & $\begin{array}{l}\text { (a) nível de mindfulness e mindful } \\
\text { eating; (b) aceitação e flexibilidade } \\
\text { psicológica; (c) estresse percebido e } \\
\text { sinais de depressão. }\end{array}$ & $\begin{array}{l}\text { Houve aumento no escore de mindful } \\
\text { eating e mindfulness, na flexibilidade } \\
\text { e aceitação psicológica. Melhora } \\
\text { nos níveis de estresse percebido e } \\
\text { depressão. }\end{array}$ \\
\hline $\begin{array}{l}\text { Cole et al. (2019) } \\
\text { EUA }^{(61)}\end{array}$ & IESTT-2; Motivation for Eating Scale. & $\begin{array}{l}\text { (a) nível de comer intuitivo; (b) peso } \\
\text { e e IMC }\end{array}$ & $\begin{array}{l}\text { Aumento significativo nos escores } \\
\text { de comer social, comer emocional, } \\
\text { permissão incondicional para comer } \\
\text { e confiança na sinalização de fome e } \\
\text { saciedade. Houve redução significativa } \\
\text { no e IMC } \text { IM. }^{\text {nativa }}\end{array}$ \\
\hline $\begin{array}{l}\text { Hanson et al. (2019) } \\
\text { Reino Unido(62) }\end{array}$ & $\begin{array}{l}\text { Whole person integrative eating } \\
\text { questionnaire }\end{array}$ & (a) comportamento alimentar; (b) peso & $\begin{array}{l}\text { Melhora significativa do comportamento } \\
\text { alimentar e maior perda de peso } \\
\text { imediata e mantida ao longo dos } 6 \\
\text { meses. }\end{array}$ \\
\hline $\begin{array}{l}\text { Stice et al. (2019) } \\
\text { EUA }^{(63)}\end{array}$ & $\begin{array}{l}\text { 20-item Thin-Ideal } \\
\text { Internalization Scale; 10-item Thin-Ideal } \\
\text { Affirmation Dissonance Scale; } 17 \text {-item } \\
\text { Satisfaction and Dissatisfaction with } \\
\text { Body Parts Scale; Positive Affect and } \\
\text { Negative Affect Scale—Revised; Eating } \\
\text { Disorder Diagnostic Interview }\end{array}$ & $\begin{array}{l}\text { (a) critérios de transtorno alimentar; (b) } \\
\text { comportamento alimentar }\end{array}$ & $\begin{array}{l}\text { No seguimento de } 6 \text { meses, } 77 \% \text { dos } \\
\text { participantes do BPT deixaram de } \\
\text { atender ao diagnóstico de transtorno } \\
\text { alimentar versus } 60 \% \text { do grupo } \\
\text { mindfulness. }\end{array}$ \\
\hline $\begin{array}{l}\text { Thomas et al. (2019) } \\
\text { EUAA }^{(64)}\end{array}$ & $\begin{array}{l}\text { DEBQ"t; Multidimensional Assessment } \\
\text { of Interoceptive Awareness; Ways of } \\
\text { Savoring Checklist. }\end{array}$ & $\begin{array}{l}\text { (a) consciência interoceptiva; } \\
\text { (b) comportamento alimentar; (c) } \\
\text { responsividade natural a recompensas }\end{array}$ & $\begin{array}{l}\text { A intervenção produziu aumento nos } \\
\text { índices de consciência interoceptiva, } \\
\text { capacidade de resposta natural às } \\
\text { recompensas, e maior redução dos } \\
\text { comportamentos alimentares externos. }\end{array}$ \\
\hline
\end{tabular}

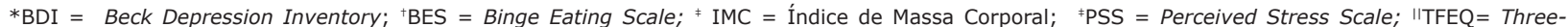
Factor Eating Questionnaire; " KIMS = Kentucky Inventory of Mindfulness Skills; **EAT-26 = Eating Attitudes Test-26; ${ }^{++} \mathrm{DEBQ}=$ Dutch Eating Behavior

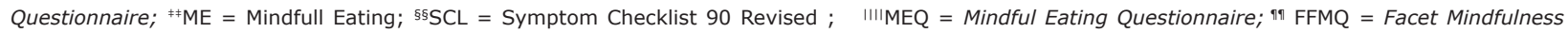
Questionnaire; ***MAAS = Mindfulness Attention Awareness Scale.

Figura 3 - Resumo da análise dos artigos incluídos: parâmetros analisados e principais resultados encontrados, Ribeirão Preto, SP, Brasil, 2019

\section{Discussão}

O objetivo do presente estudo foi realizar uma revisão integrativa do crescente número de pesquisas que investigaram o papel das intervenções baseadas em mindfulness, mindful eating e comer intuitivo na abordagem do sobrepeso, obesidade e dos TA, especialmente no que tange ao comportamento alimentar, e aos aspectos físicos e psicoemocionais. Por meio dos estudos avaliados, observou-se que tais intervenções repercutiram de forma positiva no comportamento alimentar e nos aspectos emocionais e psicológicos dos participantes; e também, em certa medida, nos aspectos físicos, no que diz respeito a perda de peso e a melhoria de parâmetros metabólicos.

O comportamento alimentar é definido(65) como atitudes relacionadas às práticas alimentares associadas a atributos socioculturais intrínsecos do indivíduo ou próprios de uma coletividade, que estejam envolvidos com o ato de se alimentar ou com o alimento em si. Nessa perspectiva, muito além de se ocupar apenas das práticas envolvidas com o alimento (qual, quanto, como, onde e com quem comemos), o comportamento alimentar envolve aspectos subjetivos em torno da alimentação, podendo ser socioculturais ou psicológicos.
À luz desse conceito, percebe-se que as intervenções centradas especialmente no ME e CI vão ao encontro do que faz parte do comportamento alimentar humano, uma vez que valorizam e consideram não apenas os aspectos biológicos, relacionados ao "o que e quanto comemos", mas também abarcam os aspectos psicossociais, emocionais e ambientais que perpassam a alimentação. Dentre seus princípios, destaca-se a reconexão com a sinalização interna de fome e saciedade e o comer guiado especialmente por esta sinalização, minimizando a ingestão alimentar em resposta a fatores emocionais ou ambientais(66). É válido ressaltar que estas intervenções não possuem como foco mudar os alimentos que o indivíduo consome, mas sim entender qual a relação que os indivíduos possuem com a comida, e sobre como a mente e o corpo entendem a experiência do comer(67). De forma especial, o ME também preconiza o não-julgamento e não-segmentação dos alimentos em "bons e ruins", auxiliando os sujeitos a compreender os motivos de suas escolhas alimentares, saindo do pilotoautomático, com abertura e gentileza(66).

A partir dessa discussão, observa-se que os resultados das intervenções observados nos estudos em relação ao comportamento alimentar guardam relação com os fundamentos do mindfulness, ME e CI. 
A atenção, presença e não-julgamento em relação a comida, e o reconhecimento da sinalização interna de fome e saciedade, são aspectos que favorecem escolhas alimentares mais flexíveis e mais consistentes com esta sinalização, minimizando o comer excessivo e o comer guiado por fatores emocionais e/ou ambientais, o que reverbera de forma positiva na relação com a comida e no comportamento alimentar (12,14-19).

O reconhecimento e a valorização do papel das emoções em nosso comer fortemente preconizado por estas intervenções, têm um papel importante na melhoria do comportamento alimentar dos sujeitos, especialmente pelo aspecto emocional com destaque para as emoções negativas, ser considerado um determinante primordial dos excessos alimentares ${ }^{(68-69)}$. Reconhecer e acolher de forma gentil e compassiva as emoções negativas, a partir da observação da própria experiência, sem julgamento ou críticas, pode evitar disfunções alimentares e o impulso de suprimir esses sentimentos por meio da comida(10). Os resultados dos estudos vão ao encontro dessa premissa, uma vez que foram obtidas melhorias no comer emocional, comer guiado por fatores externos, episódios de comer excessivo e de binge eating.

Somado a isso, os estudos em mulheres com TA, também relataram melhoria nas práticas alimentares, na relação com os alimentos e na aceitação da imagem corporal; e redução do comer emocional. Esses resultados sinalizam os impactos positivos emocionais e físicos, que podem decorrer de programas que incorporam e valorizam os princípios do $\mathrm{ME} \mathrm{e} \mathrm{CI}$, fazendo com que a perda de peso deixe de ser o único foco/ motivo de felicidade ${ }^{(9)}$.

Muito embora alguns estudos tenham realizado suas intervenções com intuito de obter redução ponderal, é importante ter em mente que as abordagens centradas no mindfulness, mindful eating e comer intuitivo não possuem este objetivo. As melhorias na relação com o comer e a comida, e os benefícios obtidos no comportamento alimentar que são preconizados nestas abordagens, podem, por consequência, cursar com diminuição no peso, mesmo não sendo este o seu foco.

A maior atenção aos sinais internos e externos que regulam a ingestão alimentar, comer mais lentamente, sentir-se satisfeito com um volume menor de alimento, e o melhor controle dos impulsos psicológicos sem a utilização da comida para satisfazer necessidades psicológicas/emocionais, pode levar à perda de peso nesse processo, mesmo não sendo este o objetivo da intervenção(70). Sob este prisma, os resultados dos estudos que não observaram superioridade das intervenções do mindfulness, ME e CI para a perda de peso não devem ser interpretados como negativos.
A prática de dietas que se centra na restrição calórica, tem sido a estratégia mais largamente utilizada para perda de peso ao longo de várias décadas(71). Todavia, acumulam-se evidências na literatura indicando a ineficácia das dietas para perda de peso, bem como a manutenção desta perda, a médio e longo prazos $^{(2-3)}$. Múltiplos mecanismos compensatórios estão envolvidos no processo de recuperação do peso que é perdido com a prática de dietas; com destaque para a intensa desconexão com a sinalização interna de fome e saciedade, uma vez que a decisão do que e do quanto comer é transferida para regras externas, gerando profundas repercussões negativas no comportamento alimentar daqueles que fazem dietas(72-73).

Nesse sentido, o comportamento alimentar dos praticantes de dieta é controlado cognitivamente, sendo necessário sempre verificar se a ingestão alimentar está de acordo com as regras externas impostas pela dieta. A desconexão com as sensações físicas de fome e saciedade acaba por desregular o comportamento alimentar dos sujeitos, fazendo com que os praticantes de dieta se tornam mais frágeis a influências ambientais, psicossociais e emocionais no comer, gerando frequentes episódios de comer excessivo e de comer emocional(73-74).

Tendo em vista que, ao contrário do preconizado pelas dietas, um dos principais objetivos das abordagens do ME e CI reside em promover melhorias no comportamento alimentar, especialmente por meio da reconexão com a sinalização interna de fome e saciedade, do não-julgamento e não-categorização dos alimentos em "permitidos e proibidos", e no estímulo ao cuidado da saúde tirando o foco do peso corporal, fica clara a contradição na utilização destas abordagens com objetivo de perda de peso.

Um ponto importante a ser considerado em relação aos estudos diz respeito a variabilidade dos protocolos de intervenção utilizados, o que dificulta a replicabilidade dos mesmos e a comparação dos resultados. Ressalta-se que os estudos, de maneira geral, não informaram aspectos inerentes aos profissionais que conduziram as intervenções, tais como a formação, o treinamento em mindfulness e a experiência clínica com a população e as práticas empregadas. Ainda, com base nos tipos de estudos analisados e em seus resultados, emerge o interesse pela dimensão experiencial dos participantes submetidos às intervenções baseadas em mindfulness, sinalizando a relevância de estudos que analisem qualitativamente a experiência do sujeito em seu próprio repertório de significados.

Apesar do interesse nas intervenções baseadas em mindful eating e comer intuitivo estar crescendo, o ano das publicações sinalizam que estas intervenções são um fenômeno recente. A maioria dos estudos publicados se concentram nos anos de 2016 e 2017, 
e o ano mais antigo de publicação é de 2010. Merece destaque o fato de que $100,0 \%$ dos estudos que compõem o corpus desta revisão são internacionais, sinalizando que, apesar dessas intervenções estarem sendo amplamente discutidas e utilizadas, o número de países produzindo sobre a temática ainda é pequeno; além de não terem sido encontrados estudos publicados sobre as abordagens no Brasil, nas bases de dados estudadas. Este apontamento traz à baila a necessidade de cautela ao generalizar esses achados para todas as populações, especialmente para indivíduos que vivem em culturas muito diversificadas, uma vez que o comportamento alimentar humano reflete interações entre o estado fisiológico, psicológico e o ambiente externo no qual se insere ${ }^{(75)}$.

A grande escassez de estudos no cenário nacional aponta para a necessidade do desenvolvimento de estudos nessa temática no país, para avançarmos em relação a sua compreensão, haja vista que essas intervenções parecem ser estratégias eficazes para auxiliar, não somente na boa relação dos indivíduos em relação à alimentação, como também para evitar comportamentos alimentares disfuncionais.

\section{Considerações Finais}

Os estudos que compõem a presente revisão oferecem uma gama de informações sobre o papel das intervenções centradas no mindfulness, ME e CI e na abordagem do sobrepeso, obesidade e dos TAs. Seus resultados sugerem que tais intervenções trazem benefícios para os sujeitos, principalmente no que diz respeito ao comportamento alimentar e aos aspectos psicoemocionais. Esses resultados estão alinhados com os princípios básicos destas intervenções, mais especificamente para o ME e CI, que se apoiam fortemente na valorização da sinalização interna de fome e saciedade como guia principal para o comer, e na abertura e não-julgamento em relação aos alimentos, o que pode minimizar episódios de comer emocional e comer excessivo; além de auxiliar na redução dos níveis de estresse e ansiedade, especialmente quando relacionados ao comer e a comida.

Mesmo não sendo o objetivo das intervenções baseadas no mindfulness, $\mathrm{ME}$ e CI, diversos estudos as utilizaram com objetivo de alcançar perda ponderal em indivíduos com excesso de peso. Analisando o corpus, observa-se que as intervenções não foram superiores, em relação ao controle, para este objetivo; o que pode, a priori, ser interpretado como um resultado negativo. Todavia, se interpretamos estes resultados à luz dos objetivos básicos das abordagens do ME e CI, percebemos que estão alinhados estes objetivos, que priorizam auxiliar os sujeitos não a perderem peso, mas a estabelecerem uma relação saudável com a comida.
A ausência de estudos no cenário brasileiro traz à tona a necessidade de que se avance nos estudos desta temática no país, considerando os resultados promissores obtidos no comportamento alimentar e na saúde emocional de indivíduos com excesso de peso e TA. Sujeitos que convivem com tais condições de saúde apresentam prejuízos em sua saúde mental e comportamentos alimentares disfuncionais, sendo ambos fonte de grande sofrimento e diminuição da qualidade de vida. Avançarmos em relação a compreensão da temática pode potencializar as abordagens especialmente as nutricionais, comumente utilizadas nestas condições. Ademais, pode também fortalecer este campo do conhecimento no país, ampliando as discussões e reflexões acerca de novas e promissoras possibilidades na abordagem do sobrepeso, obesidade e TA, que deveriam, inclusive, permear a formação profissional daqueles que irão lidar com estes sujeitos.

\section{Agradecimentos}

Agradecimento à Profa Edilaine C. da Silva Gherardi-Donato pela colaboração e incentivo na busca e aperfeiçoamento do conhecimento acerca da temática.

\section{Referências}

1. World Health Organization. Obesity and overweight. Fact sheet no. 311, 2014. [cited Dec 20, 2020]. Available from: www.who.int/mediacentre/factsheets/ fs311/en/.

2. Freire, R. Scientific evidence of diets for weight loss: Different macronutrient composition, intermittent fasting, and popular diets. Nutrition. 2020; 69:110549. doi: https://doi.org/10.1016/j.nut.2019.07.001.

3. Mann T, Tomiyama AJ, Westling E, Lew AM, Samuels B, Chatman J. Diets Are Not the Answer. Am Psychol. 2007; 62(3):220-33. doi: https://doi.org/10.1037/0003066x.62.3.220.

4. American Psychiatric Association. DSM-5: manual diagnóstico e estatístico de transtornos mentais. 5a. ed. Porto Alegre (RS): Artmed; 2014. 992p.

5. Pinzon V, Nogueira FC. Epidemiologia, curso e evolução dos transtornos alimentares. Rev Psiq Clin. 2004; 31(4):158-60. doi: http://doi.org/10.1590/ s0101-60832004000400004

6. Gracia-Arnaiz M. at bodies and thin bodies. Cultural, biomedical and market discourses on obesity. Appetite. 2010; 55(2):219-25. doi: https://doi.org/10.1016/j. appet.2010.06.002

7. Alvarenga M, Larino MA. Terapia nutricional na anorexia e bulimia nervosas. Rev Bras Psiquiatr. 2002; 24(Suppl3):39-43. doi: http://doi.org/10.1590/ S1516-44462002000700009 
8. Kabat-Zinn J. Full catastrophe living: using the wisdom of your body and mind to face stress, pain, and illness. 2a. ed. New York(NY): Delta; 1990. 720 p.

9. Dalen J, Smith BW, Shelley BM, Sloan AL, Leahigh L, Begay D. Pilot study: Mindful Eating and Living (MEAL): weight, eating behavior, and psychological outcomes associated with a mindfulness based intervention for people with obesity. Complement Ther Med. 2010; 18(6): 260-4. doi: https://doi.org/10.1016/j.ctim.2010.09.008 10. Kristeller JL, Wolever RQ. Mindfulness-based eating awareness training for treating binge eating disorder: the conceptual foundation. Eat Disord. 2011; 19(1):4961. doi: https://doi.org/10.1080/10640266.2011.5336 05

11. Kristeller JL, Wolever RQ, Sheets V. MindfulnessBased Eating Awareness Training (MB-EAT) for binge eating: a randomized clinical trial. Mindfulness. 2014; 5(3): 282-97. doi: https://doi.org/10.1007/ s12671-012-0179-1

12. Warren, JM, Smith, N, Ashwell, M. A structured literature review on the role of mindfulness, mindful eating and intuitive eating in changing eating behaviours: effectiveness and associated potential mechanisms. Nutr Res Rev. 2017; 30(2):272-83. doi: https://doi. org/10.1017/S0954422417000154

13. Tribole $E$, Resch E. Intuitive Eating. A revolutionary program that works. New York (NY): St. Martins Griffin Press; 2012. 344 p.

14. Katterman SN, Kleinman BM, Hood MM, Nackers LM, Corsica JA. Mindfulness meditation as an intervention for binge eating, emotional eating, and weight loss: A systematic review. Eat Beh. 2014; 15:197-204. https:// doi.org//10.1016/j.eatbeh.2014.01.005

15. Olson K, Emery, CF. Mindfulness and Weight Loss: A Systematic Review. Psychosomatic Medicine. 2015; 77(1):59-67. doi: https://doi.org/10.1097/ PSY.0000000000000127

16. Rogers JM, Ferrari M, Mosely K, Lang CP, Brennan $L$. Mindfulness-based interventions for adults who are overweight or obese: a meta-analysis of physical and psychological health outcomes. Obesity Rev. 2016; 18(1):51-67. doi: https://doi.org/10.1111/obr.12461

17. Carriere K, Khoury B, Gunak MM, Knauper B. Mindfulness-based interventions for weight loss: a systematic review and meta-analysis. Obesity Rev. 2017; 19(2): 164-77. doi: https://doi.org/10.1111/ obr. 12623

18. Godsey J. The role of mindfulness based interventions in the treatment of obesity and eating disorders: an integrative review. Complement Ther Med. 2013; 21(4): 430-9. doi: https://doi.org/10.1016/j. ctim.2013.06.003

19. O'Reilly GA, Cook L, Spruijt-Metz D, Black DS. Mindfulness-based interventions for obesity-related eating behaviours: a literature review. Obesity Rev. 2014; 15(6): 453- 61. doi: https://doi.org/10.1111/ obr.12156

20. Wanden-Berghe RG, Sanz-Valero J, Wanden-Berghe C. The Application of Mindfulness to Eating Disorders Treatment: A Systematic Review. Eat Disord. 2011; 19(1): 34-48. doi: https://doi.org/10.1080/10640266. 2011.533604

21. Godfrey KM, Gallo LC, Afari N. Mindfulness-based interventions for binge eating: a systematic review and meta-analysis. J Behav Med. 2015; 38(2): 348-62. doi: https://doi.org/10.1007/s10865-014-9610-5

22. Artiles RF, Staub K, Aldakak L, Eppenberger P, Ruhli $\mathrm{F}$, Bender N. Mindful eating and common diet programs lower body weight similarly: Systematic review and meta $\square$ analysis. Obesity Rev. 2019; 20(11): 1619-27. doi: https://doi.org/10.1111/obr.12918

23. Almeida CC, Assumpção AA. A eficácia do mindful eating para transtornos alimentares e obesidade: revisão integrativa. Pretextos. 2018; 3(6): 25-36.

24. Benefield LE. Implementing evidence-based practice in home care. Home Healthcare Nurse: J Home Care Hospice Prof. 2003; 21(12): 804-11. doi: https://doi. org/10.1097/00004045-200312000-00005

25. Broome ME. Integrative literature reviews for the development of concepts. In: Rodgers BL, Knafl KA, editors. Concept development in nursing: foundations, techniques and applications. Philadelphia (USA): WB Saunders; 2000. p. 231-50.

26. Mendes KDS, Silveira RCCP, Galvão CM. Revisão integrativa: método de pesquisa para a incorporação de evidências na saúde e na enfermagem. Texto Contexto- Enferm. 2008; 17(4):758-64. doi: http://doi. org/10.1590/S0104-07072008000400018

27. Moher D, Liberati A, Tetzlaff J, Altman DG. The PRISMA Group. Preferred reporting items for systematic reviews and meta-analyses: The PRISMA Statement. Annals Internal Med. 2009; 151(4):264-9. doi: https:// doi.org/10.1371/journal.pmed.1000097

28. Harris JD, Quatman E, Manring MM, Siston RA, Flanigan DC. How to write a systematic review. Am J Sports Med. 2014; 42(11):2761-8. doi: https://doi. org/10.1177/0363546513497567

29. Mason AE, Epel ES, Aschbacher K, Lustig RH, Acree $M$, Kristeller J, et al. Reduced reward-driven eating accounts for the impact of a mindfulness-based diet and exercise intervention on weight loss: Data from the SHINE randomized controlled trial. Appetite. 2016; 100(1):86-93. doi: https://doi.org/10.1016/j. appet.2016.02.009

30. Adler E, Dhruva A, Moran PJ, Daubenmier J, Acree $M$, Epel ES, et al. Impact of a Mindfulness-Based WeightLoss Intervention on Sleep Quality Among Adults with Obesity: Data from the SHINE Randomized Controlled 
Trial. J Altern Compl Med. 2017; 23(3):188-95. doi: https://doi.org/10.1089/acm.2016.0141

31. Spadaro KC, Davis KK, Sereika SM, Gibbs BB, Jakicic JM, Cohen SM. Effect of mindfulness meditation on shortterm weight loss and eating behaviors in overweight and obese adults: A randomized controlled trial. J Compl Integr Med. 2017;15(2). doi: https://doi.org/10.1515/ jcim-2016-0048

32. Stites SD, Singletary SB, Menasha A, Cooblall C, Hantula D, Axelrod S, et al. Pre-ordering lunch at work. Results of the what to eat for lunch study. Appetite. 2015; 84(1):88-97. doi: https://doi.org/10.1016/j. appet.2014.10.005

33. Duarte C, Pinto-Gouveia J, Stubbs RJ. Compassionate Attention and Regulation of Eating Behaviour: A pilot study of a brief low-intensity intervention for binge eating. Clin Psychol Psychother. 2017; 24(6):01437-047. doi: https://doi.org/10.1002/ cpp. 2094

34. Laraia BA, Adler NE, Coleman-Phox K, Vieten $C$, Mellin L, Kristeller JL, et al. Novel Interventions to Reduce Stress and Overeating in Overweight Pregnant Women: A Feasibility Study. Matern Child Health J. 2018; 22(5):670-78. doi: https://doi.org/10.1007/ s10995-018-2435-z

35. Corsica J, Hood MM, Katterman S, Kleinman B, Ivan I. Development of a novel mindfulness and cognitive behavioral intervention for stress-eating: a comparative pilot study. Eat Behav. 2014; 15(4):694-9. doi: https:// doi.org/10.1016/j.eatbeh.2014.08.002

36. Daubenmier J, Moran PJ, Kristeller J, Acree M, Bacchetti $P$, Kemeny $M E$, et al. Effects of a mindfulnessbased weight loss intervention in adults with obesity: $A$ randomized clinical trial. Obesity. 2016; 24(4):794-804. doi: https://doi.org/10.1002/oby.21396

37. Palmeira L, Pinto-Gouveia J, Cunha M. Exploring the efficacy of an acceptance, mindfulness \& compassionatebased group intervention for women struggling with their weight (Kg-Free): A randomized controlled trial. Appetite. 2017; 112(1):107-16. doi: https://doi. org/10.1016/j.appet.2017.01.027

38. Raja-Khan N, Agito K, Shah J, Stetter CM, Gustafson TS, Socolow $\mathrm{H}$, et al. Mindfulness-Based Stress Reduction in Women with Overweight or Obesity: A Randomized Controlled Trial. Obesity. 2017; 25(8):1349-59. doi: https://doi.org/10.1002/oby.21910

39. Daubenmier J, Kristeller J, Hecht FM, Maninger $N$, Kuwata M, Jhaveri $K$, et al. Mindfulness Intervention for Stress Eating to Reduce Cortisol and Abdominal Fat among Overweight and Obese Women: An Exploratory Randomized Controlled Study. J Obes. 2011; 2011:651936. doi: https://doi. org/10.1155/2011/651936
40. Daubenmier J, Lustig RH, Hecht FM, Kristeller J, Woolley J, Adam T, et al. A new biomarker of hedonic eating? A preliminary investigation of cortisol and nausea responses to acute opioid blockade. Appetite. 2014; 74(1):92-100. doi: https://doi.org/10.1016/j. appet.2013.11.014

41. Vieten C, Laraia BA, Kristeller J, Adler N, ColemanPhox K, Bush NR, et al. The mindful moms training: development of a mindfulness-based intervention to reduce stress and overeating during pregnancy. BMC Pregnancy Childbirth. 2018; 18(1):201. doi: https://doi. org/10.1186/s12884-018-1757-6

42. Boucher S, Edwards O, Gray A, Nada-Raja S, Lillis J, Tylka TL, et al. Teaching Intuitive Eating and Acceptance and Commitment Therapy Skills Via a Web-Based Intervention: A Pilot Single-Arm Intervention Study. JMIR Res Protoc. 2016; 5(4):e180. doi: https://doi. org/10.2196/resprot. 5861

43. Pinto-Gouveia J, Carvalho SA, Palmeira L, Castilho P, Duarte C, Ferreira C, et al. Incorporating psychoeducation, mindfulness and self-compassion in a new programme for binge eating (BEfree): Exploring processes of change. J Health Psychol. 2016; 24(4):46679. doi: https://doi.org/10.1177/1359105316676628 44. Alberts HJ, Mulkens S, Smeets M, Thewissen R. Coping with food cravings. Investigating the potential of a mindfulness-based intervention. Appetite. 2010; 55(1):160-3. doi: https://doi.org/10.1016/j. appet.2010.05.044

45. Courbasson CM, Nishikawa Y, Shapira LB. Mindfulness-Action Based Cognitive Behavioral Therapy for concurrent Binge Eating Disorder and Substance Use Disorders. Eat Disord. 2010; 19(1):17-33. doi: https:// doi.org/10.1080/10640266.2011.533603

46. Hepworth NS.A mindful eating group as an adjunct to individual treatment for eating disorders: a pilot study. Eat Disord. 2011; 19(1):6-16. doi: https://doi. org/10.1080/10640266.2011.533601

47. Anglin JC. Assessing the effectiveness of intuitive eating for weight loss - pilot study. Nutr. Health. 2012; 21(2):107-15. doi: https://doi. org/10.1177/0260106012459994

48. Daubenmier J, Lin J, Blackburn E, Hecht FM, Kristeller $\mathrm{J}$, Maninger $\mathrm{N}$, et al. Changes in stress, eating, and metabolic factors are related to changes in telomerase activity in a randomized mindfulness intervention pilot study. Psychoneuroendocrinology. 2012; 37(7):917-28. doi: https://doi.org/10.1016/j.psyneuen.2011.10.008 49. Woolhouse H1, Knowles A, Crafti N. Adding mindfulness to $C B T$ programs for binge eating: a mixedmethods evaluation. Eat Disord. 2012; 20(4):321-39. doi: https://doi.org/10.1080/10640266.2012.691791 50. Kidd LI, Graor CH, Murrock CJ. A mindful eating group intervention for obese women: a mixed methods 
feasibility study. Arch Psychiatr Nurs. 2013; 27(5):2118. doi: https://doi.org/10.1016/j.apnu.2013.05.004

51. Mantzios M, Giannou K. Group vs. single mindfulness meditation: exploring avoidance, impulsivity, and weight management in two separate mindfulness meditation settings. Appl Psychol Health Well Being. 2014; 6(2):173-91. doi: https://doi.org/10.1111/aphw.12023 52. Asadollahi T, Khakpour S, Ahmadi F, Seyedeh L, Tahami, Matoo $S$, et al. Effectiveness of mindfulness training and dietary regime on weight loss in obese people. J Med Life. 2015; 8(4):114-24.

53. Chacko SA, Yeh GY, Davis RB, Wee CC. A mindfulness-based intervention to control weight after bariatric surgery: Preliminary results from a randomized controlled pilot trial. Compl Ther Med. 2016; 28:13-21. doi: https://doi.org/10.1016/j.ctim.2016.07.001

54. Chung S, Zhu S, Friedmann E, Kelleher C, Kozlovsky A, Macfarlane KW, et al. Weight loss with mindful eating in African American women following treatment for breast cancer: a longitudinal study. Support Care Cancer. 2016; 24(4):1875-81. doi: https://doi.org/10.1007/ s00520-015-2984-2

55. Lucas AR, Focht BC, Cohn DE, Buckworth J, Klatt MD. A Mindfulness-Based Lifestyle Intervention for Obese, Inactive Endometrial Cancer Survivors: A Feasibility Study. Integr. Cancer Ther. 2017; 16(3):263-75. doi: https://doi.org/10.1177/1534735416668257

56. Mason AE, Epel ES, Kristeller J, Moran PJ, Dallman $M$, Lustig $\mathrm{RH}$, et al (A). Effects of a mindfulness-based intervention on mindful eating, sweets consumption, and fasting glucose levels in obese adults: data from the SHINE randomized controlled trial. J Behav Med. 2016; 39(2):201-13. doi: https://doi.org/10.1007/ s10865-015-9692-8

57. Carpenter KM, Vickerman KA, Salmon EE, Javitz HS, Epel ES, Lovejoy JC. A Randomized Pilot Study of a Phone-Based Mindfulness and Weight Loss Program. Behav Med. 2019; 45(4):271-81. doi: https://doi.org/1 0.1080/08964289.2017.1384359

58. Richards PS, Crowton S, Berrett ME, Smith $\mathrm{MH}$, Passmore K. Can patients with eating disorders learn to eat intuitively? A 2-year pilot study, Eating Disorders. 2017; 25(2):99-113. doi: https://doi.org/10.1080/106 40266.2017.1279907.

59. Kristeller JL, Jordan KD. Mindful Eating: Connecting With the Wise Self, the Spiritual Self. Front Psychol. 2018; 14;9:1271. doi: https://doi.org/10.3389/ fpsyg.2018.01271

60. Mason AE, Jhaveri K, Cohn M, Brewer JA. Testing a mobile mindful eating intervention targeting cravingrelated eating: feasibility and proof of concept. J Behav. Med. 2018; 41(2):160-173. doi: https://doi. org/10.1007/s10865-017-9884-5
61. Cole RE, Meyer SA, Newman TJ1, Kieffer AJ, Wax SG1, Stote K, et al. The My Body Knows When Program Increased Intuitive Eating Characteristics in a Military Population. Mil Med. 2019; 184(7-8):200-6. doi: https:// doi.org/10.1093/milmed/usy403

62. Hanson P, Shuttlewood E, Halder L, Shah N, Lam FT, Menon V, et al. Application of Mindfulness in a Tier 3 Obesity Service Improves Eating Behavior and Facilitates Successful Weight Loss. J Clin Endocrinol Metab. 2019; 104(3): 793-800. doi: https://doi.org/10.1210/ jc. 2018-00578

63. Stice E, Rohde P, Shaw H, Gau JM. Randomized Trial of a Dissonance-Based Group Treatment for Eating Disorders Versus a Supportive Mindfulness Group Treatment. J Consult Clin Psychol. 2019; 87(1): 79-90. doi: http://doi.org/10.1037/ccp0000365

64. Thomas EA, Mijangos JL, Hansen PA, White S, Walker D, Reimers C, et al. Mindfulness-Oriented Recovery Enhancement Restructures Reward Processing and Promotes Interoceptive Awareness in Overweight Cancer Survivors: Mechanistic Results From a Stage 1 Randomized Controlled Trial. Integr Cancer Ther. 2019; 18:1534735419855138. doi: https://doi. org/10.1177/1534735419855138

65. Garcia RW. Representações sociais da alimentação e saúde e suas repercussões no comportamento alimentar. Physis: Rev Saúde Coletiva. 1997; 7(2):51-68. doi: http://doi.org/10.1590/S0103-73311997000200004

66. Rossy L. The mindfulness-based eating solution. Oakland (CA): New Harbinger Publications; 2016. 224 p. 67. Camilleri GM, Méjean C, Bellisle F, Andreeva VA, Kesse-Guyot E, Hercberg $S$, et al. Intuitive Eating Dimensions Were Differently Associated with Food Intake in the General Population-Based NutriNet-Santé Study. J Nutr. 2016; 147(1):61-9. doi: https://doi.org/10.3945/ jn.116.234088

68. Gibson EL. Emotional influences on food choice: Sensory, physiological and psychological pathways. Physiol Behav. 2006; 89(1):53-61. doi: https://doi. org/10.1016/j.physbeh.2006.01.024

69. Canetti L, Bachar E, Berry EM. Food and emotion. Behavioural Proc. 2002; 60(2):157-64. doi: https://doi. org/10.1016/S0376-6357(02)00082-7

70. Kabat-Zinn J. Mindfulness $\square$ based interventions in context: past, present, and future. Clin Psychol Sci Pract. 2003; 10(2):144-56. doi: https://doi.org/10.1093/ clipsy.bpg016

71. Foxcroft L. A tirania das dietas. São Paulo(SP): Três Estrelas; 2013. 280 p.

72. Alvarenga M, Palacow V, Scagliusi F. Dieta e seus efeitos no comportamento alimentar. In: Alvarenga M, Figueiredo M, Timerman F., editores. Nutrição Comportamental. São Paulo(SP): Manole; 2015. p. 69-100. 
73. Stroebe W, Van Koningsbruggen GM. Why Most Dieters Fail but Some Succeed: A Goal Conflict Model of Eating Behavior. Psychol Rev. 2013; 120(1):110-38. doi: https://doi.org/10.1037/a0030849

74. Herman $\mathrm{CP}$, Polivy JA. boundary model for the regulation of eating. In: Stunkard JA, Stellar E, editors. Eating and its disorders. New York (NY): Raven Press; 1984. p.141-56.

75. Quaioti TCB, Almeida SS. Determinantes psicobiológicos do comportamento alimentar: uma ênfase em fatores ambientais que contribuem para a obesidade. Psicol USP. 2006; 17(4): 193-211. doi: https://doi.org/10.1590/S0103-65642006000400011

\section{Contribuição dos autores}

Concepção e planejamento do estudo: Marina Rodrigues Barbosa e Fernanda Rodrigues de Oliveira Penaforte. Obtenção dos dados: Marina Rodrigues Barbosa e Fernanda Rodrigues de Oliveira Penaforte. Análise e interpretação dos dados: Marina Rodrigues Barbosa, Fernanda Rodrigues de Oliveira Penaforte e Ana Flavia De Sousa Silva. Análise estatística: Marina Rodrigues Barbosa, Fernanda Rodrigues de Oliveira Penaforte e Ana Flavia De Sousa Silva. Redação do manuscrito: Marina Rodrigues Barbosa, Fernanda Rodrigues de Oliveira Penaforte e Ana Flavia De Sousa Silva. Revisão crítica do manuscrito: Marina Rodrigues Barbosa.

Todos os autores aprovaram a versão final do texto.

Conflito de interesse: os autores declararam que não há conflito de interesse.
Autor correspondente:

Marina Rodrigues Barbosa

E-mail: marinarbarbosarb@gmail.com

(D) https://orcid.org/0000-0001-7502-243X
Recebido: 26.01.2020

Aceito: 29.07 .2020
Copyright $\odot 2020$ SMAD, Rev. Eletrônica Saúde Mental Álcool Drog. Este é um artigo de acesso aberto distribuído sob os termos da Licença Creative Commons CC BY-NC.

Esta licença permite que outros remixem, adaptem e criem a partir do seu trabalho para fins não comerciais, e embora os novos trabalhos tenham de lhe atribuir o devido crédito e não possam ser usados para fins comerciais, os usuários não têm de licenciar esses trabalhos derivados sob os mesmos termos. 